\title{
METRIC ABSTRACT ELEMENTARY CLASSES WITH PERTURBATIONS
}

\author{
ÅSA HIRVONEN AND TAPANI HYTTINEN
}

\begin{abstract}
We define an abstract setting suitable for investigating perturbations of metric structures generalizing the notion of a metric abstract elementary class. We show how perturbation of Hilbert spaces with an automorphism fit into this framework, where the perturbations are built into the definition of the class being investigated. Further, assuming homogeneity and some other properties true in the Hilbert example, we develop a notion of independence for this setting and show that it satisfies the usual independence axioms. Finally we define an isolation notion. Although it remains open whether this isolation gives any reasonable form of primeness, we prove that dominance works.
\end{abstract}

\section{INTRODUCTION}

In metric model theory there are examples of classes which come down in stability hierarchy if instead of the usual metric way of measuring stability one measures stability up to perturbations. We will take a closer look at one such example. The question we try to answer in this paper is: Can one use $\omega$-stability up to perturbations to prove better behaviour of the independence and constructible models compared to what one gets from the usual metric stability or superstability. The answer will be yes. We also want to remind the reader that in stable classes there tends to be only one independence notion i.e. any two are the same over the sets over which both behave well. One example of this phenomenon will be proved.

In [9] we introduced an abstract approach for studying metric structures, by defining a metric abstract elementary class as a metric version of Shelah's abstract elementary classes. In this paper we continue the work by giving an abstract approach to perturbations of metric structures, presented by Ben Yaacov in [1] and [2]. Our approach is to add classes $\mathbb{F}_{\varepsilon}$ of so-called $\varepsilon$-isomorphisms, which closely resemble the perturbation mappings of [2].

Date: March 5, 2010.

2000 Mathematics Subject Classification. Primary 03C52; Secondary 03C45.

Key words and phrases. metric structures, perturbation.

$\AA$. Hirvonen supported by the Graduate School in Mathematical Logic and Algebra (MALJA) and the Finnish Academy of Science and Letters (Vilho, Yrjö and Kalle Väisälä foundation). T. Hyttinen partially supported by the Academy of Finland, grant 1106753. 
This gives a very natural approach to perturbations as built into the framework instead of seen as a later modification of the structures. The abstract framework also gives a natural approach to unbounded structures, avoiding the somewhat cumbersome "emboundment" that Ben Yaacov introduces in [1] in order to be able to perturb the norm of Banach spaces. Also it seems that compactness (in the sense of metric model theory) is of very limited value when everything is done only up to perturbations, this remains to be seen.

As in [9] we assume a countable Löwenheim-Skolem number (where size naturally is measured by the density character), the existence of arbitrarily large models, joint embedding, amalgamation and homogeneity for Galois types (i.e. with respect to genuine isomorphisms). In addition we assume a form of amalgamation also for the $\varepsilon$-isomorphisms and a stronger perturbation property which links the perturbation mappings to Galois types. The topology we consider on the type space is given by a metrizable uniformity $\mathbf{d}^{p}$. This resembles the distance arising in Ben Yaacov's work by combining perturbation and the moving of realizations of types. In our setting it, however, also includes the moving of parameters, so our notion of $\omega$-stability is weaker than " $\omega$-stability up to perturbation" in [2].

A natural example of a metric abstract elementary class with perturbations (although not presented here in further detail) is the class of all Banach spaces, where the perturbation mappings are Banach-space isomorphisms (i.e. linear homeomorphisms). Then the amalgamation property is given by Kislyakov's construction, described in [7] (and in [9] in the setting of a metric abstract elementary class). It may be of interest to people in functional analysis, that the monster model constructed using this form of amalgamation has the property, that any Banach-space isomorphism between two small enough spaces extends to a Banach-space automorphism of the monster with the same norm. Further the perturbation property in this class arises from the fact that in the monster model finite tuples have the same almost isometric type if and only if they have the same Galois type.

To be able to develop model theoretical tools, we will further need a form of $\omega$-stability and a property which we call completeness of type-spaces. As an example of a class satisfying these further assumptions we show how to put Ben Yaacov's and Berenstein's Hilbert spaces with generic automorphisms ([3]) into our setting. This class also satisfies the assumption 
of models being good (see Definition 2.19). This assumption can sometimes replace the assumption of completeness of type-spaces and can be used to prove that separable $\omega$ - $\mathbf{d}^{p}$-saturated models are almost isomorphic (Definition 5.4), a result obtained by Ben Yaacov for continuous logic with perturbations in [1, Proposition 2.7].

Having established a framework for structures with perturbation we develop an independence notion based on splitting and show that this independence notion satisfies the usual independence axioms (to the extent natural for a metric structure). Using this independence notion we also show that $\omega$-d $\mathbf{d}^{p}$-stable structures with complete type-spaces are $\lambda$-d $\mathbf{d}^{p}$-stable for all $\lambda$ and further that $\omega$-d $\mathbf{d}^{p}$-stability and complete type spaces or good models imply ordinary stability in all $\lambda=\lambda^{\aleph_{0}}$.

Finally we introduce a notion of isolation. This enables us to define primary sets, but the question whether these are prime in any reasonable sense remains open. However, we do prove dominance for these primary sets.

In section 2 we introduce the framework and present our notational conventions. Section 3 shows how the Hilbert example sits into the framework. In section 4 we investigate the $\mathbf{d}^{p}$ and present two ways of finding limit types. Section 5 presents the concept of $\omega-\mathbf{d}^{p}$-saturation, which corresponds to Ben Yaacov's $\mathfrak{p}$-approximate $\aleph_{0}$-saturation. We see how to find $\omega$-d $\mathbf{d}^{p}$-saturated sets and prove the almost isomorphism result mentioned above. Section 6 defines splitting and the independence notion based on it. Here we prove that the independence axioms hold and see how our stability and independence notions relate to the standard ones in homogeneous model theory. Finally section 7 deals with isolation.

The authors would like to thank Hans-Olav Tylli for many helpful discussions on Hilbert spaces and their operators.

\section{Generalizing metric abstract elementary Classes}

When reading the following definitions one may keep in mind the following example: (in section 3 we give the main example): Let $\mathbb{K}$ be the class of all Banach spaces and let $\preccurlyeq$ be the closed subspace relation. $f: \mathcal{A} \rightarrow \mathcal{B}$ is in $\mathbb{F}_{\varepsilon}$ if $f$ is an onto linear isomorphism in the functional analysis sense (i.e. a linear homeomorphism) with $\|f\|$ and $\left\|f^{-1}\right\|$ at most $e^{\varepsilon}$, where $e=\lim _{n \rightarrow \infty}\left(1+\frac{1}{n}\right)^{n}$. The exponential is used in order for the epsilons to behave nicely when composing functions, see Definition 2.2. 
As in [9] we investigate a class of complete metric space structures of a fixed countable vocabulary. The structures are many-sorted and of the form

$$
\mathcal{M}=\left\langle\mathcal{A}_{0}, \mathcal{A}_{1}, \ldots, \mathbb{R}, \mathrm{d}_{0}, \mathrm{~d}_{1}, \ldots, c_{0}, c_{1}, \ldots, R_{0}, R_{1}, \ldots, F_{0}, F_{1}, \ldots\right\rangle,
$$

where

(1) each $\mathcal{A}_{i}$ is a complete metric space with metric $\mathrm{d}_{i}$ (with values in $\mathbb{R})$

(2) $\mathbb{R}$ is an isomorphic copy of the ordered field of real numbers

$$
(\mathbb{R},+, \cdot, 0,1, \leq,|\cdot|),
$$

(3) each $c_{i}$ is a constant and each $R_{i}$ a relation,

(4) each $F_{i}$ is a function $F_{i}: \mathcal{B}_{0} \times \cdots \times \mathcal{B}_{m} \rightarrow \mathcal{B}_{m+1}$ where $\mathcal{B}_{j} \in$ $\left\{\mathcal{A}_{0}, \ldots, \mathcal{A}_{n-1}, \mathbb{R}\right\}$.

We will use some shorthand notation. When we talk about elements of a model $\mathcal{M}$ we mean an element of some sort of $\mathcal{M} . a \in \mathcal{M}$ refers to a finite tuple of elements of $\mathcal{M}$. For a set $A, \bar{A}$ refers, depending on whether we are inside a model or not, to either the sortwise metric closure of $A$ or some completion of $A$. Since size is measured with respect to density character, $|A|=\operatorname{dens}(A)$. For $\mathcal{M}$ a many-sorted model, $|\mathcal{M}|$ is the sum of the density characters of its sorts. The cardinality of a set $A$ is denoted card $(A)$.

Both models and their domains will be denoted by $\mathcal{A}, \mathcal{B}$ etc., $A, B$ etc. refers to sets not necessary being models. After we construct a monster model $\mathfrak{M}$, models will be submodels of $\mathfrak{M}$ with size small enough compared to $|\mathfrak{M}|$. Also by $A \subset \mathfrak{M}$ we mean that $A$ is a small enough subset. (The notion of small enough will become apparent when we define $\mathfrak{M}$.)

We consider the distance of two finite tuples $\mathrm{d}(a, b)$, where $a$ and $b$ have the same length, to be the maximum of their coordinatewise distances. When $A$ and $B$ are finite sets of the same cardinality we consider them to be ordered and hence by $\mathrm{d}(A, B)$ mean this maximum of coordinatewise distances for a fixed ordering.

In [9] MAECs were defined as follows:

Definition 2.1. We call a class $\left(\mathbb{K}, \preccurlyeq_{\mathbb{K}}\right)$ of $\tau$-structures for some fixed vocabulary $\tau$ a metric abstract elementary class, $M A E C$, if the following hold:

(1) Both $\mathbb{K}$ and the binary relation $\preccurlyeq_{\mathbb{K}}$ are closed under isomorphism.

(2) If $\mathcal{A} \preccurlyeq_{\mathbb{K}} \mathcal{B}$ then $\mathcal{A}$ is a substructure of $\mathcal{B}$ (i.e. each sort of $\mathcal{A}$ is a substructure of the corresponding sort of $\mathcal{B})$.

$(3) \preccurlyeq_{\mathbb{K}}$ is a partial order on $\mathbb{K}$. 
(4) If $\left(\mathcal{A}_{i}\right)_{i<\delta}$ is a $\preccurlyeq \mathbb{K}$-increasing chain then there is a model $\overline{\bigcup_{i<\delta} \mathcal{A}_{i}}$, unique up to the choice of completion such that

(a) $\overline{\bigcup_{i<\delta} \mathcal{A}_{i}} \in \mathbb{K}$

(b) for each $j<\delta, \mathcal{A}_{j} \preccurlyeq \mathbb{K} \overline{\bigcup_{i<\delta} \mathcal{A}_{i}}$.

In addition if each $\mathcal{A}_{i} \preccurlyeq \mathbb{K} \mathcal{B} \in \mathbb{K}$ and the completion is the metric closure in $\mathcal{B}$ then $\overline{\bigcup_{i<\delta} \mathcal{A}_{i}} \preccurlyeq_{\mathbb{K}} \mathcal{B}$.

(5) If $\mathcal{A}, \mathcal{B}, \mathcal{C} \in \mathbb{K}, \mathcal{A} \preccurlyeq_{\mathbb{K}} \mathcal{C}, \mathcal{B} \preccurlyeq_{\mathbb{K}} \mathcal{C}$ and $\mathcal{A} \subseteq \mathcal{B}$ then $\mathcal{A} \preccurlyeq_{\mathbb{K}} \mathcal{B}$.

(6) There exists a Löwenheim-Skolem number $\operatorname{LS}^{\mathrm{d}}(\mathbb{K})$ such that if $\mathcal{A} \in$ $\mathbb{K}$ and $A \subset \mathcal{A}$ then there is $B \supseteq A$ such that $|B|=|A|+\operatorname{LS}^{\mathrm{d}}(\mathbb{K})$ and $\bar{B} \preccurlyeq_{\mathbb{K}} \mathcal{A}$.

We generalize this by loosening the requirements of being an isomorphism and add a class $\mathbb{F}$ of homeomorphisms to play the role of isomorphisms.

Definition 2.2. $\left(\mathbb{K}, \preccurlyeq, \mathbb{F}_{\varepsilon}\right)_{\varepsilon \geq 0}$ is a metric abstract elementary class with perturbations if

$(1)(\mathbb{K}, \preccurlyeq)$ is a MAEC.

(2) the $\mathbb{F}_{\varepsilon}$ are collections of bijective mappings between members of $\mathbb{K}$ such that:

(a) $\mathbb{F}_{\delta} \subseteq \mathbb{F}_{\varepsilon}$ for $\delta<\varepsilon, \mathbb{F}_{0}=\bigcap_{\varepsilon>0} \mathbb{F}_{\varepsilon}$ and $\mathbb{F}_{0}$ is the collection of genuine isomorphisms between models in $\mathbb{K}$,

(b) for all $\varepsilon>0$, all members of $\mathbb{F}_{\varepsilon}$ satisfy a common modulus of uniform continuity, i.e., there is a function $\Delta^{\varepsilon}:(0, \infty) \rightarrow(0, \infty)$ such that for any $f: \mathcal{A} \rightarrow \mathcal{B}$ with $f \in \mathbb{F}_{\varepsilon}$, any $x, y \in \mathcal{A}$, and any $\delta>0$,

$$
\text { if } \mathrm{d}(x, y)<\Delta^{\varepsilon}(\delta) \text { then } \mathrm{d}(f(x), f(y)) \leq \delta,
$$

(c) if $f \in \mathbb{F}_{\varepsilon}$ then $f^{-1} \in \mathbb{F}_{\varepsilon}$,

(d) if $f \in \mathbb{F}_{\varepsilon}, g \in \mathbb{F}_{\delta}$ and $\operatorname{dom}(g)=\operatorname{rng}(f)$ then $g \circ f \in \mathbb{F}_{\varepsilon+\delta}$.

(e) if $\left(f_{i}\right)_{i<\alpha}$ is an increasing chain of $\varepsilon$-isomorphisms, i.e $f_{i} \in \mathbb{F}_{\varepsilon}$, $f_{i}: \mathcal{A}_{i} \rightarrow \mathcal{B}_{i}, \mathcal{A}_{i} \preccurlyeq \mathcal{A}_{i+1}, \mathcal{B}_{i} \preccurlyeq \mathcal{B}_{i+1}$ and $f_{i} \subseteq f_{i+1}$ for all $i<\alpha$, then there is an $\varepsilon$-isomorphism $f: \overline{\bigcup_{i<\alpha} \mathcal{A}_{i}} \rightarrow \overline{\bigcup_{i<\alpha} \mathcal{B}_{i}}$ such that $f\left\lceil\mathcal{A}_{i}=f_{i}\right.$ for all $i<\alpha$.

Definition 2.3 ( $\varepsilon$-isomorphisms and -embeddings). If $\varepsilon \geq 0$, we refer to the mappings $f \in \mathbb{F}_{\varepsilon}$ as $\varepsilon$-isomorphisms. Correspondingly $\varepsilon$-automorphisms are $\varepsilon$-isomorphisms $f: \mathcal{A} \rightarrow \mathcal{A}$ for some $\mathcal{A}$. If $\mathcal{A}, \mathcal{B} \in \mathbb{K}$ and $f: \mathcal{A} \rightarrow \mathcal{B}$ is a mapping such that $f: \mathcal{A} \rightarrow f(\mathcal{A})$ is an $\varepsilon$-isomorphism and $f(\mathcal{A}) \preccurlyeq \mathcal{B}$, then $f$ is called an $\varepsilon$-embedding. Here $f(\mathcal{A})$ means the image of $\mathcal{A}$ with the structure induced by the inclusion mapping $f(\mathcal{A}) \hookrightarrow \mathcal{B}$. 
It is worth noting that if $f: \mathcal{A} \rightarrow \mathcal{B}$ is an $\varepsilon$-isomorphism and $\mathcal{A}^{\prime} \preccurlyeq \mathcal{A}$ then $f \uparrow \mathcal{A}^{\prime}$ need not be an $\varepsilon$-embedding.

Definition 2.4 (Joint embedding property). $\mathbb{K}$ is said to have the joint embedding property if for any $\mathcal{A}, \mathcal{B} \in \mathbb{K}$ there are $\mathcal{C} \in \mathbb{K}$ and 0-embeddings $f: \mathcal{A} \rightarrow \mathcal{C}$ and $g: \mathcal{B} \rightarrow \mathcal{C}$.

Remark 2.5. Note that since a MAEC is closed under 0-isomorphisms, when applying the joint embedding property we may assume that one of the embeddings is the identity mapping. However, with $\varepsilon$-embeddings we need to be more careful, since they do not necessary preserve $\preccurlyeq$-submodels.

Definition 2.6 (Amalgamation property). $\mathbb{K}$ is said to have the amalgamation property if whenever $\mathcal{A}, \mathcal{B}, \mathcal{C} \in \mathbb{K}, \mathcal{A} \preccurlyeq \mathcal{B}$ and $f: \mathcal{A} \rightarrow \mathcal{C}$ is an $\varepsilon$-embedding then there are $\mathcal{B}^{\prime}, \mathcal{C}^{\prime} \in \mathbb{K}$ with $\mathcal{B}^{\prime} \succcurlyeq \mathcal{B}, \mathcal{C}^{\prime} \succcurlyeq \mathcal{C}$ and an $\varepsilon$ embedding $g: \mathcal{B}^{\prime} \rightarrow \mathcal{C}^{\prime}$ with $g \supseteq f$.

Remark 2.7. Note that, since 0 -embeddings preserve $\preccurlyeq$-submodels, this version implies the ordinary amalgamation property: if $\mathcal{A} \preccurlyeq \mathcal{B}$ and $\mathcal{A} \preccurlyeq \mathcal{C}$ then there is $\mathcal{D} \in \mathbb{K}$ with $\mathcal{D} \succcurlyeq \mathcal{C}$ and a 0 -embedding $f: \mathcal{B} \rightarrow \mathcal{D}$ with $f \uparrow \mathcal{A}=\mathrm{id}$.

We also have the following:

Lemma 2.8. Assuming the amalgamation property, if $\mathcal{A}^{\prime}, \mathcal{A} \in \mathbb{K}, \mathcal{A}^{\prime} \preccurlyeq \mathcal{A}$ and $f: \mathcal{A}^{\prime} \rightarrow \mathcal{A}$ is an $\varepsilon$-embedding, then there are $\mathcal{B}, \mathcal{D} \in \mathbb{K}$ with $\mathcal{A} \preccurlyeq \mathcal{B} \preccurlyeq \mathcal{D}$ and an $\varepsilon$-embedding $g: \mathcal{B} \rightarrow \mathcal{D}$ with $g \supseteq f$.

Proof. By the amalgamation property there are $\mathcal{B} \succcurlyeq \mathcal{A}$ and $\mathcal{C} \succcurlyeq \mathcal{A}$ and an $\varepsilon$-embedding $g^{\prime}: \mathcal{B} \rightarrow \mathcal{C}$ with $g^{\prime} \supseteq f$. Then we may use standard amalgamation (with 0 -embeddings) and amalgamate $\mathcal{B}$ and $\mathcal{C}$ over $\mathcal{A}$. So we get $\mathcal{D} \succcurlyeq \mathcal{B}$ and a 0 -embedding $h: \mathcal{C} \rightarrow \mathcal{D}$ with $h \uparrow \mathcal{A}=$ id. Then $g=h \circ g^{\prime}$ is an $\varepsilon$-embedding of $\mathcal{B}$ into $\mathcal{D}$ and $g \uparrow \mathcal{A}^{\prime}=h \circ g^{\prime} \uparrow \mathcal{A}^{\prime}=h \circ f=f$.

Definition 2.9 (Galois-type in a model). For $\mathcal{A}, \mathcal{B} \in \mathbb{K}$ and $\left\{a_{i}: i<\right.$ $\alpha\} \subset \mathcal{A},\left\{b_{i}: i<\alpha\right\} \subset \mathcal{B}$ we say that $\left(a_{i}\right)_{i<\alpha}$ and $\left(b_{i}\right)_{i<\alpha}$ have the same Galois-type in $\mathcal{A}$ and $\mathcal{B}$ respectively,

$$
\mathrm{t}^{g}\left(\left(a_{i}\right)_{i<\alpha} / \emptyset ; \mathcal{A}\right)=\mathrm{t}^{g}\left(\left(b_{i}\right)_{i<\alpha} / \emptyset ; \mathcal{B}\right)
$$

if there are $\mathcal{C} \in \mathbb{K}$ and 0-embeddings $f: \mathcal{A} \rightarrow \mathcal{C}$ and $g: \mathcal{B} \rightarrow \mathcal{C}$ such that $f\left(a_{i}\right)=g\left(b_{i}\right)$ for every $i<\alpha$. 
The amalgamation property ensures that having the same Galois-type is a transitive relation. It it also true that elements have the same Galois-type in a model and its $\preccurlyeq$-extensions and that 0 -embeddings preserve Galois-types.

Definition 2.10 (0-homogeneity). We call $\mathbb{K}$ 0-homogeneous or Galoishomogeneous if whenever $\mathcal{A}, \mathcal{B} \in \mathbb{K},\left\{a_{i}: i<\alpha\right\} \subset \mathcal{A},\left\{b_{i}: i<\alpha\right\} \subset \mathcal{B}$ and for all $n<\omega, i_{0}, \ldots, i_{n-1}<\alpha$

$$
\mathrm{t}^{g}\left(\left(a_{i_{0}}, \ldots, a_{i_{n-1}}\right) / \emptyset ; \mathcal{A}\right)=\mathrm{t}^{g}\left(\left(b_{i_{0}}, \ldots, b_{i_{n-1}}\right) / \emptyset ; \mathcal{B}\right)
$$

then

$$
\mathrm{t}^{g}\left(\left(a_{i}\right)_{i<\alpha} / \emptyset ; \mathcal{A}\right)=\mathrm{t}^{g}\left(\left(b_{i}\right)_{i<\alpha} / \emptyset ; \mathcal{B}\right)
$$

With the properties defined so far we can construct a 0-homogeneous, $\varepsilon$-model-homogeneous monster model.

Theorem 2.11. Let $\left(\mathbb{K}, \preccurlyeq, \mathbb{F}_{\varepsilon}\right)_{\varepsilon \geq 0}$ be a metric abstract elementary class with perturbations satisfying the joint embedding property, the amalgamation property and 0-homogeneity. Let $\mu$ be a given infinite cardinal. Then there is $\mathfrak{M} \in \mathbb{K}$ satisfying:

(1) ( $\mu$-universality): $\mathfrak{M}$ is $\mu$-universal, i.e. for all $\mathcal{A} \in \mathbb{K}$ with $|\mathcal{A}|<\mu$ there is a 0 -embedding $f: \mathcal{A} \rightarrow \mathfrak{M}$.

(2) ( $\mu$-0-homogeneity): If $\left(a_{i}\right)_{i<\alpha}$ and $\left(b_{i}\right)_{i<\alpha}$ are sequences of elements of $\mathfrak{M}$ such that $\alpha<\mu$ and for all $n<\omega$ and $i_{0}, \ldots, i_{n-1}<\alpha$

$$
\mathrm{t}^{g}\left(\left(a_{i_{0}}, \ldots, a_{i_{n-1}}\right) / \emptyset ; \mathfrak{M}\right)=\mathrm{t}^{g}\left(\left(b_{i_{0}}, \ldots, b_{i_{n-1}}\right) / \emptyset ; \mathfrak{M}\right)
$$

then there is a 0-automorphism $f$ of $\mathfrak{M}$ such that $f\left(a_{i}\right)=b_{i}$ for all $i<\alpha$.

(3) ( $\mu-\varepsilon$-model-homogeneity): If $\mathcal{A} \preccurlyeq \mathfrak{M},|\mathcal{A}|<\mu$ and there is an $\varepsilon$ embedding $f: \mathcal{A} \rightarrow \mathfrak{M}$ then there is an $\varepsilon$-automorphism of $\mathfrak{M}$ extending $f$.

Proof. Let $\mu$ be given and choose a regular $\kappa>\mu^{\aleph_{0}}$. We define models $\mathcal{A}_{\alpha}$ for $\alpha<\kappa$ inductively as follows: When $\alpha=0$, we use the joint embedding property to construct a $\mu$-universal model $\mathcal{A}_{0}$. When $\alpha=\delta$ is a limit, we let $\mathcal{A}_{\delta}=\overline{\bigcup_{\beta<\delta} \mathcal{A}_{\beta}}$. When $\alpha=\beta+1$ we take care of properties (2) and (3) by an inner induction. Let $\left(f_{i}^{\beta}\right)_{i<\kappa_{\beta}}$ list all partial mappings $f: \mathcal{A}_{\beta} \rightarrow \mathcal{A}_{\beta}$ satisfying the property that for all finite $b \in \operatorname{dom}(f)$

$$
\mathrm{t}^{g}\left(b / \emptyset ; \mathcal{A}_{\beta}\right)=\mathrm{t}^{g}\left(f(b) / \emptyset ; \mathcal{A}_{\beta}\right)
$$


Further let $\left(g_{i}^{\beta}\right)_{i<\kappa_{\beta}}$ list all mappings $g$ such that $g: \mathcal{A}^{\prime} \rightarrow \mathcal{A}_{\beta}$ is an $\varepsilon$ embedding for some $\varepsilon>0$ and $\mathcal{A}^{\prime} \preccurlyeq \mathcal{A}_{\beta}$. Then we define an increasing chain of models $\mathcal{B}_{i}, i<\kappa_{\beta}$, such that

- $\mathcal{B}_{0}=\mathcal{A}_{\beta}$,

- $\mathcal{B}_{\delta}=\overline{\bigcup_{i<\delta} \mathcal{B}_{i}}$, when $\delta<\kappa_{\beta}$ is a limit,

- there is a 0-embedding $F_{i}^{\beta}: \mathcal{A}_{\beta} \rightarrow \mathcal{B}_{i+1}$ extending $f_{i}^{\beta}$,

- if $g_{i}^{\beta}$ is an $\varepsilon$-embedding $\mathcal{A}^{\prime} \rightarrow \mathcal{A}_{\beta}$ then there is some $\mathcal{B}^{\prime}$ with $\mathcal{B}_{i} \preccurlyeq$ $\mathcal{B}^{\prime} \preccurlyeq \mathcal{B}_{i+1}$ and an $\varepsilon$-embedding $G_{i}^{\beta}: \mathcal{B}^{\prime} \rightarrow \mathcal{B}_{i+1}$ extending $g_{i}^{\beta}$.

When constructing $\mathcal{B}_{i+1}$ first note that by 0 -homogeneity and the fact that Galois-types are preserved under $\preccurlyeq$-extensions there are $\mathcal{B}_{i+1}^{\prime}$ and 0 embeddings $f: \mathcal{A}_{\beta} \rightarrow \mathcal{B}_{i+1}^{\prime}$ and $g: \mathcal{B}_{i} \rightarrow \mathcal{B}_{i+1}^{\prime}$ such that for all $b \in \operatorname{dom}\left(f_{i}^{\beta}\right)$, $f(b)=g \circ f_{i}^{\beta}$ and as in 2.5 we may assume $g$ is the identity. Hence $f$ gives the required $F_{i}^{\beta}$. Next note that given $g_{i}^{\beta}$ as above $\mathcal{A}^{\prime} \preccurlyeq \mathcal{A}_{\beta} \preccurlyeq \mathcal{B}_{i} \preccurlyeq \mathcal{B}_{i+1}^{\prime}$, so by Lemma 2.8 there are $\mathcal{B}_{i+1} \succcurlyeq \mathcal{B}^{\prime} \succcurlyeq \mathcal{B}_{i+1}^{\prime}$ and an $\varepsilon$-embedding $G_{i}^{\beta}: \mathcal{B}^{\prime} \rightarrow \mathcal{B}_{i+1}$ extending $g_{i}^{\beta}$.

Hence we can define $\mathcal{B}_{i}$ for all $i<\kappa_{\beta}$ and then let $\mathcal{A}_{\alpha}=\overline{\bigcup_{i<\kappa_{\beta}} \mathcal{B}_{i}}$.

Finally let $\mathfrak{M}=\bigcup_{i<\kappa} \mathcal{A}_{i}$ and note that since $\operatorname{cf}(\kappa)>\omega, \mathfrak{M}=\overline{\mathfrak{M}}$. Then clearly $\mathfrak{M}$ is $\mu$-universal and to see that properties (2) and (3) hold note that inverses of type-preserving mappings (0-embeddings) are type-preserving and inverses of $\varepsilon$-embeddings are $\varepsilon$-embeddings. Hence any given embedding with dom $<\mu$ can be extended by a back-and-forth argument to an automorphism of $\mathfrak{M}$. Here we use property (2e) of Definition 2.2 to continue the back-and-forth construction through limits.

As usual, from now on we will work inside a monster model $\mathfrak{M}$ as in 2.11 for some large enough $\mu$. Then when we write $A \subset \mathfrak{M}$ or $\mathcal{A} \preccurlyeq \mathfrak{M}$ this will include the assumption that $A$ or $\mathcal{A}$ has size (i.e. density character) less than $\mu$.

Notation 2.12. We denote by $\operatorname{Aut}_{\varepsilon}(\mathfrak{M} / A)$ the set of $\varepsilon$-automorphisms of $\mathfrak{M}$ fixing $A$ pointwise.

Definition 2.13 (Galois-type). We say that $\left(a_{i}\right)_{i<\alpha}$ and $\left(b_{i}\right)_{i<\alpha}$ have the same Galois-type over $A, \mathrm{t}^{g}\left(\left(a_{i}\right)_{i<\alpha} / A\right)=\mathrm{t}^{g}\left(\left(b_{i}\right)_{i<\alpha} / A\right)$, if there is $f \in$ $\operatorname{Aut}_{0}(\mathfrak{M} / A)$ such that $f\left(a_{i}\right)=b_{i}$ for every $i<\alpha$.

Note that for $A=\emptyset$ the above definition coincides with the notion of Galois-type in $\mathfrak{M}$ in Definition 2.9 .

In [9] we introduced the perturbation property, to achieve what the Perturbation lemma gives in [8]. Here we will define an even stronger version 
of perturbation tying our $\varepsilon$-isomorphisms and Galois-types together. The role of this property will be discussed in section 4 .

We define a nonnegative real-valued function $\mathbf{d}^{p}$ on the set of pairs of Galois-types of finite tuples over the empty set.

Definition 2.14. For $a, b \in \mathfrak{M}$ and $\varepsilon>0$ we write

$$
\mathbf{d}^{p}\left(\mathrm{t}^{g}(a / \emptyset), \mathrm{t}^{g}(b / \emptyset)\right) \leq \varepsilon
$$

if there are $\varepsilon$-automorphisms $f$ and $g$ of $\mathfrak{M}$ such that $\mathrm{d}(f(a), b) \leq \varepsilon$ and $\mathrm{d}(g(b), a) \leq \varepsilon$.

The perturbation property then tells us that this function vanishes on $(p, q)$ exactly when $p=q$ :

Definition 2.15 (Perturbation property). Assume $\mathbb{K}$ satisfies the joint embedding property, the amalgamation property and 0-homogeneity so that $\mathfrak{M}$ can be constructed. Then $\mathbb{K}$ is said to have the perturbation property if whenever $a, b \in \mathfrak{M}$ are such that $\mathbf{d}^{p}\left(\mathrm{t}^{g}(a / \emptyset), \mathrm{t}^{g}(b / \emptyset)\right)=0$ (i.e. $\mathbf{d}^{p}\left(\mathrm{t}^{g}(a / \emptyset), \mathrm{t}^{g}(b / \emptyset)\right) \leq \varepsilon$ for all positive $\left.\varepsilon\right)$ then $\mathrm{t}^{g}(a / \emptyset)=\mathrm{t}^{g}(b / \emptyset)$.

We are now ready to list the assumptions we will make on $\mathbb{K}$.

Assumption 2.16. From now on we will assume that $\left(\mathbb{K}, \preccurlyeq, \mathbb{F}_{\varepsilon}\right)_{\varepsilon \geq 0}$ is a metric abstract elementary class with perturbations satisfying the following:

- $\operatorname{LS}^{\mathrm{d}}(\mathbb{K})=\omega$,

- $\mathbb{K}$ has arbitrarily large models,

- $\mathbb{K}$ has the joint embedding property 2.4 ,

- $\mathbb{K}$ has the amalgamation property 2.6,

- $\mathbb{K}$ is 0 -homogeneous as defined in 2.10 ,

- $\mathbb{K}$ has the perturbation property 2.15

Hence we can construct a $\mu$-universal, $\mu$-0-homogeneous and $\mu$ - $\varepsilon$-modelhomogeneous monster model $\mathfrak{M}$ for some $\mu$ larger than any cardinality we will encounter and consider only $\preccurlyeq$-submodels of $\mathfrak{M}$.

Beside these assumptions we will mostly need to assume that the type space is complete with respect to $\mathbf{d}^{p}$ (actually with respect to a metrizable uniformity defined by $\mathbf{d}^{p}$, see section 4 ), more specifically:

Definition 2.17. We say that $\mathbb{K}$ has complete type-spaces if for all finite sets $A \mathbf{d}^{p}$-Cauchy sequences of types over $A$ have a limit, i.e. if $\left(a_{i}\right)_{i<\omega}$ is a 
sequence with the property that for all $\varepsilon>0$ there is $n_{0}<\omega$ such that for all $m, n \geq n_{0}$

$$
\mathbf{d}^{p}\left(\mathrm{t}^{g}\left(a_{m} A / \emptyset\right), \mathrm{t}^{g}\left(a_{n} A / \emptyset\right)\right)<\varepsilon,
$$

then there exists some $a$ with the property that for all $\varepsilon>0$ there is $n_{0}<\omega$ such that for all $n>n_{0}$

$$
\mathbf{d}^{p}\left(\mathrm{t}^{g}(a A / \emptyset), \mathrm{t}^{g}\left(a_{n} A / \emptyset\right)\right)<\varepsilon .
$$

In some constructions the assumption of completeness of type-spaces can be replaced by the assumption of models being good (i.e. the pair $(\mathcal{A}, \mathcal{A})$ is good).

Definition 2.18. If $a \in \mathcal{A}$ and $b \in \mathcal{B}$, we write

$$
\mathbf{d}_{\varepsilon}\left(\mathrm{t}^{g}(a / \emptyset ; \mathcal{A}), \mathrm{t}^{g}(b / \emptyset ; \mathcal{B})\right)<\delta
$$

if there are $\mathcal{A}^{\prime} \succcurlyeq \mathcal{A}$ and $\mathcal{B}^{\prime} \succcurlyeq \mathcal{B}$ and an $\varepsilon$-embedding $f: \mathcal{A}^{\prime} \rightarrow \mathcal{B}^{\prime}$ such that $\mathrm{d}(f(a), b)<\delta$.

We then say that $f: A \rightarrow \mathcal{B}$, for $A \subset \mathcal{A}$ is a weak $\varepsilon$-embedding if for all $a \in A, \mathbf{d}_{\varepsilon}\left(\mathrm{t}^{g}(a / \emptyset ; \mathcal{A}), \mathrm{t}^{g}(f(a) / \emptyset ; \mathcal{B})\right)<\delta$ for all $\delta>0$.

Definition 2.19. Assume $A \subseteq \mathcal{A}$. We say that the pair $(A, \mathcal{A})$ is good if for all $\varepsilon>0$ there is some $\delta>0$ such that if $f: A \rightarrow \mathcal{B}$ is a weak $\delta$-embedding then there are $\mathcal{A}^{\prime} \succcurlyeq \mathcal{A}, \mathcal{B}^{\prime} \succcurlyeq \mathcal{B}$ and an $\varepsilon$-embedding $g: \mathcal{A}^{\prime} \rightarrow \mathcal{B}^{\prime}$ such that $g \supseteq f$. (Note that $g\lceil\mathcal{A}$ need not be an $\varepsilon$-embedding.)

Definition 2.20. When $A \subseteq \mathfrak{M}$, we say that $A$ is good in $\mathfrak{M}$ if for all $\varepsilon>0$ there is some $\delta>0$ such that for all weak $\delta$-embeddings $f: A \rightarrow \mathfrak{M}$ there is $g \in \operatorname{Aut}_{\varepsilon}(\mathfrak{M})$ such that $g \supseteq f$.

Lemma 2.21. Let $\mu$ be as in the construction of $\mathfrak{M}$. Assume $A \subset \mathfrak{M}$ and $|A|<\mu$. Then $A$ is good in $\mathfrak{M}$ if and only if $(A, \mathcal{A})$ is good for some $\mathcal{A} \preccurlyeq \mathfrak{M}$ with $A \subseteq \mathcal{A}$ and $|\mathcal{A}|<\mu$. Especially $\mathcal{A} \preccurlyeq \mathfrak{M}$, with $|\mathcal{A}|<\mu$, is good in $\mathfrak{M}$ if and only if $(\mathcal{A}, \mathcal{A})$ is good.

Proof. First note that if $|\mathcal{A}|<\mu$ and a given mapping extends to an $\varepsilon$ embedding $\mathcal{A} \preccurlyeq \mathcal{A}^{\prime} \rightarrow \mathcal{B}^{\prime}$, we may always assume $\left|\mathcal{A}^{\prime}\right|,\left|\mathcal{B}^{\prime}\right|<\mu$, since we by the assumption $\mathrm{LS}^{\mathrm{d}}=\omega$ and a back-and-forth construction may find such small enough models (assuming $\mu>\aleph_{0}$ ). Hence if $A$ is good in $\mathfrak{M},(A, \mathcal{A})$ is good for any $\mathcal{A} \supset A$ in $\mathfrak{M}$. On the other hand, if $(A, \mathcal{A})$ is good for some small enough $\mathcal{A}$, and $f: \mathcal{A}^{\prime} \rightarrow \mathcal{B}^{\prime}$ is and $\varepsilon$-embedding extending some given weak embedding of $A$, we may assume $\mathcal{A}^{\prime}, \mathcal{B}^{\prime}$ are small enough and hence 
inside $\mathfrak{M}$ (by universality). Then $f$ has an extension to an automorphism inside $\mathfrak{M}$.

In this context the natural notion of stability will be with respect to $\mathbf{d}^{p}$. However, for this we first need to generalize the definition.

Definition 2.22. For $a, b \in \mathfrak{M}$ and $A \subset \mathfrak{M}$, we define

$$
\mathbf{d}^{p}\left(\mathrm{t}^{g}(a / A), \mathrm{t}^{g}(b / A)\right)=\sup \left\{\mathbf{d}^{p}\left(\mathrm{t}^{g}(a c / \emptyset), \mathrm{t}^{g}(b c / \emptyset)\right): c \in A \text { finite }\right\} .
$$

Then stability is defined in the natural way.

Definition 2.23. We say that $\mathbb{K}$ is $\lambda$-d $\mathbf{d}^{p}$-stable if for any set $A$ with $|A| \leq \lambda$, the set of types over $A$ has density $\lambda$ with respect to $\mathbf{d}^{p}$, i.e. looking at the situation inside $\mathfrak{M}$, there is a set $A^{*}$ of cardinality $\lambda$ such that for any $a \in \mathfrak{M}$ and $\varepsilon>0$ there is some $a^{\prime} \in A^{*}$ such that $\mathbf{d}^{p}\left(\mathrm{t}^{g}(a / A), \mathrm{t}^{g}\left(a^{\prime} / A\right)\right)<\varepsilon$.

In section 4 we will see that $\mathbf{d}^{p}$ defines a metrizable uniformity on the type space and that the above definition hence makes sense.

\section{Hilbert SPACES With AN AUTOMORPhism}

We now turn to an example of a MAEC with perturbations investigated in [6] and [3] in the continuous setting as an example of a theory of continuous logic that is not $\omega$-stable but $\omega$-stable up to perturbation (see e.g. [1]). The idea is, that you allow arbitrarily small perturbations of parts of the language of the structure. In the setting of metric abstract elementary classes with perturbations, these perturbations are built into the $\mathbb{F}_{\varepsilon}$ 's.

Definition 3.1. Let $\mathbb{K}_{H}$ be the class of complex Hilbert spaces equipped with an automorphism $\tau$, i.e. $\tau$ is a surjective unitary operator. We then define $\left(H^{\prime}, \tau^{\prime}\right) \preccurlyeq(H, \tau)$ as the ordinary submodel relation i.e. the $\preccurlyeq$-submodels of $(H, \tau)$ are closed subspaces closed under $\tau$.

When $\varepsilon \geq 0$ we let $f:\left(H_{1}, \tau_{1}\right) \rightarrow\left(H_{2}, \tau_{2}\right)$ belong to $\mathbb{F}_{\varepsilon}$ if $f$ is an isometric isomorphism between the Hilbert spaces $H_{1}$ and $H_{2}$ that additionally satisfies

$$
\left\|\tau_{1}-f^{-1} \tau_{2} f\right\| \leq \varepsilon
$$

and

$$
\left\|\tau_{1}^{-1}-f^{-1} \tau_{2}^{-1} f\right\| \leq \varepsilon
$$

where $\|\cdot\|$ is the operator norm defined by $\|T\|=\sup _{\|x\|=1}\|T(x)\|$. 
It is worth noting, that Ben Yaacov, Berenstein, Usvyatsov and Zadka consider only generic automorphisms. We define here our class as covering all automorphisms $\tau$, but the restriction to generic ones also constitute an example class and both classes produce the same monster model. Our demands on the $\varepsilon$-isomorphisms arise from the form of perturbation Ben Yaacov and Berenstein use in [3]: they define an r-perturbation of $\left(H_{0}, \tau_{0}\right)$ to $\left(H_{1}, \tau_{1}\right)$ to be an isometric isomorphism $U: H_{0} \cong H_{1}$ satisfying in addition $\left\|U \tau_{0} U^{-1}-\tau_{1}\right\| \leq r$. This in turn is connected to a criterion by Ben Yaacov, Usvyatsov and Zadka for an automorphism being generic and the Weyl-von Neumann-Berg theorem. To state these results we need the definitions of some spectra of a bounded operator.

Definition 3.2. Consider a Hilbert space $H$ and a bounded operator $T$ on $H$. Then we can define the spectrum, the point spectrum and the essential spectrum of $T$ by:

$$
\begin{aligned}
\sigma(T)= & \{\lambda \in \mathbb{C}: T-\lambda I \text { is not invertible }\}, \\
\sigma_{p}(T)= & \{\lambda \in \mathbb{C}: \operatorname{ker}(T-\lambda I) \neq 0\}, \\
\sigma_{e}(T)= & \{\text { non-isolated points of } \sigma(T)\} \cup \\
& \{\lambda \in \mathbb{C}: \operatorname{dim} \operatorname{ker}(T-\lambda I)=\infty\} .
\end{aligned}
$$

Fact 3.3. (Ben Yaacov, Usvyatov, Zadka [6]) Let $H$ be a Hilbert space and let $\tau$ be a unitary operator on $H$. Then $(H, \tau)$ is existentially closed as a model of the continuous first order theory of infinite dimensional Hilbert spaces with an automorphism if and only if $\sigma(\tau)=S^{1}$ where $S^{1}$ is the unit circle.

Definition 3.4. Let $H$ be a Hilbert space and let $T_{0}, T_{1}$ be bounded operators on $H$. Then $T_{0}$ and $T_{1}$ are approximately unitarily equivalent if there is a sequence of unitary operators $\left(U_{n}\right)_{n<\omega}$ such that $\left\|T_{1}-U_{n} T_{0} U_{n}^{*}\right\| \rightarrow 0$.

Fact 3.5. (Weyl-von Neumann-Berg Theorem) Let $H$ be a separable Hilbert space and let $T_{0}, T_{1}$ be normal operators on $H$. Then $T_{0}$ and $T_{1}$ are approximately unitarily equivalent if and only if

(1) $\sigma_{e}\left(T_{0}\right)=\sigma_{e}\left(T_{1}\right)$

(2) $\operatorname{dim} \operatorname{ker}\left(T_{0}-\lambda I\right)=\operatorname{dim} \operatorname{ker}\left(T_{1}-\lambda I\right)$ for all $\lambda \in \mathbb{C} \backslash \sigma_{e}\left(T_{0}\right)$.

This characterization of approximately unitarily equivalent spaces is the key point in the proof of $\omega$-stability up to perturbation, see [3] and [6]. 
Fact 3.6. (Ben Yaacov, Berenstein, Usvyatsov, Zadka) The continuous first order theory of infinite dimensional Hilbert spaces with a generic automorphism is $\omega$-stable up to perturbation of the automorphism.

Remark 3.7. Henson and Iovino observed that the above theory is not $\omega$-stable in the ordinary metric sense (see [11]) i.e. with respect to the distance defined as the infimum of distances of realizations of types.

We next turn to the task of proving that the class defined in Definition 3.1 indeed satisfies the assumptions in 2.16.

Lemma 3.8. The class $\left(\mathbb{K}_{H}, \preccurlyeq\right)$ is a metric abstract elementary class with Löwenheim-Skolem number $\aleph_{0}$, having arbitrarily large models and satisfying joint embedding and 0-homogeneity.

Proof. It is trivial to see that $\left(\mathbb{K}_{H}, \preccurlyeq\right)$ forms a MAEC with $\mathrm{LS}^{\mathrm{d}}=\aleph_{0}$ and arbitrarily large models satisfying 0-homogeneity since the class is axiomatizable in continuous first order logic and joint embedding is taken care of by orthogonal direct sums.

Lemma 3.9. The collections $\mathbb{F}_{\varepsilon}$ defined in 3.1 satisfy the properties of Definition 2.2.

Proof. This is easily seen remembering that in this example $\varepsilon$-isomorphisms are isometries of the underlying Hilbert spaces.

Lemma 3.10. The class defined in 3.1 has the amalgamation property.

Proof. Assume $\left(H^{\prime}, \tau^{\prime}\right) \preccurlyeq(H, \tau)$ and that $f:\left(H^{\prime}, \tau^{\prime}\right) \rightarrow\left(H_{1}, \tau_{1}\right)$ is an $\varepsilon$ embedding. Then we may write $(H, \tau)=\left(H^{\prime}, \tau^{\prime}\right) \oplus\left(H^{\prime \prime}, \tau^{\prime \prime}\right)$ and embed $(H, \tau)$ into $\left(H_{1}, \tau_{1}\right) \oplus\left(H^{\prime \prime}, \tau^{\prime \prime}\right)$ with some extension of $f$.

Since the amalgamation property holds, we have a monster model for the class and may consider our last property, perturbation.

Lemma 3.11. The class defined in 3.1 has the perturbation property.

Proof. Let $a, b \in \mathfrak{M}$ be such that $\mathbf{d}^{p}\left(\mathrm{t}^{g}(a / \emptyset), \mathrm{t}^{g}(b / \emptyset)\right)=0$, i.e. for all $\varepsilon>0$ there are $\varepsilon$-automorphisms of $\mathfrak{M}$ mapping $a \varepsilon$-close to $b$ and vice versa. Now consider the spaces spanned by $\left\{\tau^{k} a: k \in \mathbb{Z}\right\}$ and $\left\{\tau^{k} b: k \in \mathbb{Z}\right\}$ respectively. The assumption implies that any finite part $\left\{\tau^{k} a:-m<k<m\right\}$ can be mapped arbitrarily close to the corresponding set $\left\{\tau^{k} b:-m<k<m\right\}$ 
by an isometric isomorphism of the base Hilbert space. Hence the Hilbert spaces

$$
\overline{\operatorname{Span}\left\{\tau^{k} a: k \in \mathbb{Z}\right\}} \text { and } \overline{\operatorname{Span}\left\{\tau^{k} b: k \in \mathbb{Z}\right\}}
$$

are isometrically isomorphic and since these spaces are closed under $\tau$ they are $\preccurlyeq$-submodels of $\mathfrak{M}$. Further, the function mapping each $\tau^{k} a$ to $\tau^{k} b$ makes no error in mapping $\tau$, so the isomorphism is a 0 -isomorphism of $\left(\mathbb{K}_{H}, \preccurlyeq, \mathbb{F}_{\varepsilon}\right)_{\varepsilon \geq 0}$ and hence extends to a 0 -automorphism of $\mathfrak{M}$.

Collecting the results, we have:

Theorem 3.12. The class of Hilbert spaces with an automorphism, with $\preccurlyeq$ defined as the ordinary submodel relation and the classes $\mathbb{F}_{\varepsilon}$ defined as in 3.1 forms a metric abstract elementary class satisfying the assumptions in 2.16.

We also have both completeness of type-spaces and goodness:

Theorem 3.13. $\mathbb{K}_{H}$ has complete type-spaces.

Proof. This follows from the fact that the class of Hilbert spaces with an automorphism is axiomatizable in continuous first order logic and hence closed under ultraproducts (see [4]). Now let $A$ be finite and let $\left(a_{i}\right)_{i<\omega}$ be a sequence such that $\left(\mathrm{t}^{g}\left(a_{i} / A\right)\right)_{i<\omega}$ is $\mathbf{d}^{p}$-Cauchy. By switching to a subsequence if necessary, we may assume that $\mathbf{d}^{p}\left(\mathrm{t}^{g}\left(a_{j} / A\right), \mathrm{t}^{g}\left(a_{i} / A\right)\right) \leq 2^{-i}$ for all $i<j<\omega$. Then let $f_{j i}$ be a $2^{-i}$ automorphism witnessing this, i.e. satisfying $\mathrm{d}\left(f_{j i}\left(a_{j} A\right), a_{i} A\right) \leq 2^{-i}$. Let $A \cup\left\{a_{i}: i<\omega\right\} \subset \mathcal{A} \preccurlyeq \mathfrak{M}$ such that $\mathcal{A}$ is closed under each $f_{j i}$. Then let $D$ be an ultrafilter on $\omega$ extending the Frechet filter and consider the ultrapower $(\mathcal{A})_{D}$. Then the diagonal embeddings of each $a_{i} A$ still form a $\mathbf{d}^{p}$-Cauchy sequence, witnessed by the functions $\left(\left(f_{j i}\right)_{n<\omega}\right) / D$, which are $2^{-i}$-automorphisms of $(\mathcal{A})_{D}$.

Now the sequence has a $\mathbf{d}^{p}$-limit, namely $a=\left(\left(a_{i}\right)_{i<\omega}\right) / D$, and this is seen by considering the mappings

$$
F_{i}=\left(\left(f_{j i}\right)_{j<\omega}\right) / D
$$

where we let $f_{j i}=$ id if $j \leq i$. Since the componentwise mappings of $F_{i}$ are isometric automorphisms of the base Hilbert space of $\mathcal{A}$ and the componentwise error in mapping $\tau$ is at most $2^{-i}, F_{i}$ is an $2^{-i}$-automorphism of $(\mathcal{A})_{D}$. Further it moves the diagonal embedding of $A$ by at most $2^{-i}$ showing that $\mathbf{d}^{p}\left(\mathrm{t}^{g}(a / A), \mathrm{t}^{g}\left(a_{i} / A\right)\right) \leq 2^{-i}$.

Theorem 3.14. If $\mathcal{A} \in \mathbb{K}_{H}$ then $(\mathcal{A}, \mathcal{A})$ is good as defined in 2.19 
Proof. Let $\mathcal{A} \in \mathbb{K}_{H}$ and $\varepsilon>0$ be given. We choose $\delta=\varepsilon /(2+\sqrt{8})$ and show that if $f: \mathcal{A} \rightarrow \mathcal{B}$ is a weak $\delta$-embedding, then there are $\mathcal{A}^{\prime} \succcurlyeq \mathcal{A}$ and $\mathcal{B}^{\prime} \succcurlyeq \mathcal{B}$ and an $\varepsilon$-embedding $g: \mathcal{A}^{\prime} \rightarrow \mathcal{B}^{\prime}$ extending $f$.

So let $f: \mathcal{A} \rightarrow \mathcal{B}$ be a weak $\delta$-embedding, i.e. for all (finite tuples) $a \in \mathcal{A}$ and $\delta^{\prime}>0$ there are $\mathcal{A}_{1} \succcurlyeq \mathcal{A}$ and $\mathcal{B}_{1} \succcurlyeq \mathcal{B}$ and a $\delta$-embedding $f_{1}$ : $\mathcal{A}_{1} \rightarrow \mathcal{B}_{1}$ such that $\mathrm{d}\left(f_{1}(a), f(a)\right)<\delta^{\prime}$. Since $\delta$-embeddings are isometric isomorphisms of the underlying Hilbert space, we see that weak embeddings must also be isometric isomorphisms of the Hilbert space. Further for any $a \in \mathcal{A}$ with $\|a\|=1$ and any $\delta^{\prime}>0$ there is a $\delta$-embedding $f_{1}$ for the tuple $\left(a, \tau_{\mathcal{A}}(a), \tau_{\mathcal{A}}^{-1}(a)\right)$ as above and hence (noting that $\tau_{\mathcal{A}}=\tau_{\mathcal{A}^{\prime}} \uparrow \mathcal{A}$ and similarly for $\left.\tau_{\mathcal{B}}\right)$

$$
\begin{aligned}
\left\|\left(f \tau_{\mathcal{A}}-\tau_{\mathcal{B}} f\right)(a)\right\| \leq & \left\|\left(f \tau_{\mathcal{A}}-f_{1} \tau_{\mathcal{A}}\right)(a)\right\|+\left\|\left(f_{1} \tau_{\mathcal{A}}-\tau_{\mathcal{B}^{\prime}} f_{1}\right)(a)\right\| \\
& +\left\|\left(\tau_{\mathcal{B}^{\prime}} f_{1}-\tau_{\mathcal{B}^{\prime}} f\right)(a)\right\| \\
\leq & \delta^{\prime}+\delta+\delta^{\prime}
\end{aligned}
$$

and similarly for $\tau^{-1}$. Hence weak embeddings are isometric isomorphisms of the underlying Hilbert space satisfying the norm requirements for embeddings but whose images are not necessarily submodels of the target model, i.e. closed under $\tau$. Since $f$ is an isometric isomorphism of the Hilbert space, we however know, that $f(\mathcal{A})$ must be a closed subspace of $\mathcal{B}$, so writing $\mathcal{B}=\left(H_{\mathcal{B}}, \tau_{\mathcal{B}}\right)$ we may write $H_{\mathcal{B}}=H \oplus H^{\prime}$ where $H=\operatorname{rng}(f)$.

We now define $\mathcal{A}^{\prime}=\mathcal{B}^{\prime}=\mathcal{A} \oplus \mathcal{B}$, (i.e. $\left(H_{\mathcal{A}}, \tau_{\mathcal{A}}\right) \oplus\left(H_{\mathcal{B}}, \tau_{\mathcal{B}}\right)$ ). Clearly $\mathcal{A} \preccurlyeq \mathcal{A}^{\prime}$ and $\mathcal{B} \preccurlyeq \mathcal{B}^{\prime}$ so we just need to find an $\varepsilon$-embedding $f^{\prime}: \mathcal{A}^{\prime} \rightarrow \mathcal{B}^{\prime}$ extending $f$. But here we just define $f^{\prime} \uparrow \mathcal{A}=f, f^{\prime} \uparrow H=f^{-1}$ and $f^{\prime} \uparrow H^{\prime}=\mathrm{id}$. Then $f^{\prime}$ is an isometric isomorphism of the underlying Hilbert spaces and to see that it satisfies $\left\|\tau_{\mathcal{A}^{\prime}}-f^{\prime-1} \tau_{\mathcal{B}^{\prime}} f^{\prime}\right\| \leq \varepsilon$ (we prove only the first condition since $\left\|\tau_{\mathcal{A}^{\prime}}^{-1}-f^{\prime-1} \tau_{\mathcal{B}^{\prime}}^{-1} f^{\prime}\right\| \leq \varepsilon$ can be proved similarly) we first consider the parts separately:

Case 1: If $a \in H_{\mathcal{A}}$ and $\|a\|=1$ then since $f^{\prime} \uparrow \mathcal{A}=f$ we have

$$
\left\|f^{\prime} \tau_{\mathcal{A}^{\prime}}(a)-\tau_{\mathcal{B}^{\prime}} f^{\prime}(a)\right\|=\left\|f \tau_{\mathcal{A}}(a)-\tau_{\mathcal{B}} f(a)\right\| \leq \delta .
$$

Case 2: If $b \in H$, and $\|b\|=1$, then $b=f(a)$ for some $a \in \mathcal{A}$ with $\|a\|=1$ and $\left\|f \tau_{\mathcal{A}}(a)-\tau_{\mathcal{B}} f(a)\right\| \leq \delta$. Hence

$$
\begin{aligned}
\left\|f^{\prime} \tau_{\mathcal{A}^{\prime}}(b)-\tau_{\mathcal{B}^{\prime}} f^{\prime}(b)\right\| & =\left\|f^{\prime} \tau_{\mathcal{B}}(b)-\tau_{\mathcal{A}} f^{\prime}(b)\right\| \\
& =\left\|f^{\prime} \tau_{\mathcal{B}} f(a)-f^{\prime} f \tau_{\mathcal{A}}(a)\right\| \\
& =\left\|\tau_{\mathcal{B}} f(a)-f \tau_{\mathcal{A}}(a)\right\| \\
& \leq \delta .
\end{aligned}
$$


Case 3: If $b \in H^{\prime}$, and $\|b\|=1$, then since $\tau_{\mathcal{A}^{\prime}}(b)=\tau_{\mathcal{B}}(b) \in \mathcal{B}$, there are $x \in H$ and $y \in H^{\prime}$ such that $\tau_{\mathcal{A}^{\prime}}(b)=x+y$. Then

$$
\left\|f^{\prime} \tau_{\mathcal{A}^{\prime}}(b)-\tau_{\mathcal{B}^{\prime}} f^{\prime}(b)\right\|=\left\|f^{\prime}(x+y)-(x+y)\right\| \leq\left\|f^{\prime}(x)\right\|+\|x\|=\sqrt{2}\|x\| .
$$

Now $\tau_{\mathcal{A}^{\prime}}^{-1}(x+y)=b \in H^{\prime}$, so $\operatorname{Pr}_{H^{\prime}}\left(\tau_{\mathcal{A}^{\prime}}^{-1}(x)\right)+\operatorname{Pr}_{H^{\prime}}\left(\tau_{\mathcal{A}^{\prime}}^{-1}(y)\right)=b$. Further $\left\|\operatorname{Pr}_{H^{\prime}}\left(\tau_{\mathcal{A}^{\prime}}^{-1}(y)\right)\right\| \leq\|y\|$ and since $\tau_{\mathcal{A}^{\prime}}^{-1}(x)$ is $\delta\|x\|$-close to something in $H$, $\left\|\operatorname{Pr}_{H^{\prime}}\left(\tau_{\mathcal{A}^{\prime}}^{-1}(x)\right)\right\| \leq \delta\|x\|$. Hence we have $1=\|b\| \leq \delta\|x\|+\|y\|$ but by definition of $x$ and $y, 1=\|b\|^{2}=\|x\|^{2}+\|y\|^{2}$. Hence

$$
1-\|x\|^{2}=\|y\|^{2} \geq(1-\delta\|x\|)^{2}
$$

and hence

$$
\|x\| \leq \frac{2 \delta}{\delta^{2}+1} \leq 2 \delta
$$

Combining this with the result above, we have

$$
\left\|f^{\prime} \tau_{\mathcal{A}^{\prime}}(b)-\tau_{\mathcal{B}^{\prime}} f^{\prime}(b)\right\| \leq \sqrt{2}\|x\| \leq \sqrt{8} \delta .
$$

Finally, if $a \in \mathcal{A}^{\prime}$ is arbitrary, there are $x \in \mathcal{A}, y \in H$ and $z \in H^{\prime}$ such that $a=x+y+z$. Then

$$
\begin{aligned}
\left\|\left(f^{\prime} \tau_{\mathcal{A}^{\prime}}-\tau_{\mathcal{B}^{\prime}} f^{\prime}\right)(a)\right\| \leq & \left\|\left(f^{\prime} \tau_{\mathcal{A}^{\prime}}-\tau_{\mathcal{B}^{\prime}} f^{\prime}\right)(x)\right\|+\left\|\left(f^{\prime} \tau_{\mathcal{A}^{\prime}}-\tau_{\mathcal{B}^{\prime}} f^{\prime}\right)(y)\right\| \\
& +\left\|\left(f^{\prime} \tau_{\mathcal{A}^{\prime}}-\tau_{\mathcal{B}^{\prime}} f^{\prime}\right)(z)\right\| \\
\leq & \delta\|x\|+\delta\|y\|+\sqrt{8} \delta\|z\| \\
\leq & (2+\sqrt{8})\|a\| \leq \varepsilon\|a\| .
\end{aligned}
$$

Theorem 3.15. $\mathbb{K}_{H}$ is $\omega$-d $\mathbf{d}^{p}$-stable. Actually, if $A \subset \mathfrak{M}$ is separable, then there is a countable set $A^{*} \subset \mathfrak{M}$ such that for any $a \in \mathfrak{M}$ and $\varepsilon>0$ there is some $a^{\prime} \in A^{*}$ and an $\varepsilon$-automorphism $f$ of $\mathfrak{M}$ such that $f \uparrow A=\mathrm{id}$ and $\mathrm{d}\left(f(a), a^{\prime}\right)<\varepsilon$.

Proof. This is proved by imitating Ben Yaacov's and Berenstein's proof of Fact 3.6. Let $A \subset \mathfrak{M}$ be separable. Then we may consider a separable $\mathcal{A} \preccurlyeq \mathfrak{M}$ containing $A$ and by universality of $\mathfrak{M}$ another separable $\mathcal{B} \preccurlyeq \mathfrak{M}$ such that $\tau_{\mathcal{B}}$ has full spectrum and $\mathcal{A} \cap \mathcal{B}=\{0\}$. Now consider $\mathcal{C}=\mathcal{A} \oplus \mathcal{B}$. We claim that any dense (countable) subset of $\mathcal{C}$ will do as our $A^{*}$. So let $a \in \mathfrak{M}$. If $a \in \mathcal{A}$, there is nothing to prove. Otherwise consider $d=$ $a-P_{\mathcal{A}}(a)$, where $P_{\mathcal{A}}$ is the orthogonal projection onto $\mathcal{A}$. By universality of $\mathfrak{M}$, let $\mathcal{D}$ be a separable model containing $d$ with $\mathcal{A} \cap \mathcal{D}=\{0\}$ and such that $\tau_{\mathcal{D}}$ has full spectrum. Fixing an isometric isomorphism of the Hilbert spaces of $\mathcal{B}$ and $\mathcal{D}$ and using Fact 3.5 we may for any $\varepsilon>0$ find an $\varepsilon$-isomorphism 
$f: \mathcal{D} \rightarrow \mathcal{B}$. Then $\operatorname{id}_{\mathcal{A}} \oplus f: \mathcal{A} \oplus \mathcal{D} \rightarrow \mathcal{A} \oplus \mathcal{B}$ belongs to $\mathbb{F}_{\varepsilon}$ and extends to an $\varepsilon$-automorphism of $\mathfrak{M}$ and $\left(\operatorname{id}_{\mathcal{A}} \oplus f\right)\left(P_{\mathcal{A}}(a)+d\right)$ is the desired realization in $\mathcal{C}$.

Remark 3.16. By considerations like the ones in the proof above we see that all $(H, \tau)$ where $\tau$ is generic (i.e. has full spectrum) are $\omega$-d $\mathbf{d}^{p}$-saturated, see Definition 5.1.

\section{TYPES AND $\mathbf{d}^{p}$}

Recall the definition of $\mathbf{d}^{p}$ (the version below is what we get by the perturbation property):

Definition 4.1. For $a, b \in \mathfrak{M}$ and $\varepsilon \geq 0, \mathbf{d}^{p}\left(\mathrm{t}^{g}(a / \emptyset), \mathrm{t}^{g}(b / \emptyset)\right) \leq \varepsilon$ if there are $\varepsilon$-automorphisms $f$ and $g$ of $\mathfrak{M}$ such that $\mathrm{d}(f(a), b) \leq \varepsilon$ and $\mathrm{d}(g(b), a) \leq$ $\varepsilon$.

For $a, b \in \mathfrak{M}$ and $A \subset \mathfrak{M}$,

$$
\mathbf{d}^{p}\left(\mathrm{t}^{g}(a / A), \mathrm{t}^{g}(b / A)\right)=\sup \left\{\mathbf{d}^{p}\left(\mathrm{t}^{g}(a c / \emptyset), \mathrm{t}^{g}(b c / \emptyset)\right): c \in A \text { finite }\right\} .
$$

It is easy to see that the definition only depends on the Galois-types of $a$ and $b$. Also, the perturbation property (together with 0-homogeneity) ensures that if $\mathbf{d}^{p}\left(\mathrm{t}^{g}(a / A), \mathrm{t}^{g}(b / A)\right)=0$ then $a$ and $b$ do have the same Galois-type over $A$ (proved below). However, it is worth noting, that when $\mathbf{d}^{p}\left(\mathrm{t}^{g}(a / A), \mathrm{t}^{g}(b / A)\right)$ is strictly positive, we do not have a function fixing $A$ pointwise that moves $a$ near $b$.

Lemma 4.2. If $a, b \in \mathfrak{M}, A \subset \mathfrak{M}$ and $\mathbf{d}^{p}\left(\mathrm{t}^{g}(a / A), \mathrm{t}^{g}(b / A)\right)=0$ then $\mathrm{t}^{g}(a / A)=\mathrm{t}^{g}(b / A)$.

Proof. By 0-homogeneity it is enough to show that for any finite $c \in A$, $\mathrm{t}^{g}(a c / \emptyset)=\mathrm{t}^{g}(b c / \emptyset)$ which is equal to the demand $\mathrm{t}^{g}(a / c)=\mathrm{t}^{g}(b / c)$. But since our assumption asserts that $\mathbf{d}^{p}\left(\mathrm{t}^{g}(a / A), \mathrm{t}^{g}(b / A)\right)=0$, i.e.

$$
\sup \left\{\mathbf{d}^{p}\left(\mathrm{t}^{g}(a c / \emptyset), \mathrm{t}^{g}(b c / \emptyset)\right): c \in A \text { finite }\right\}=0,
$$

this is exactly what the perturbation property gives us.

Thus for any $A, \mathbf{d}^{p}$ is a semimetric on $S(A)$. To investigate the behavior of $\mathbf{d}^{p}$ let us first have a look at the moduli of uniform continuity $\Delta^{\varepsilon}$ from $2 \mathrm{~b}$ of Definition 2.2. From the demands on the classes $\mathbb{F}_{\varepsilon}$ we can easily deduce:

Fact 4.3. (1) If $\delta<\varepsilon$ then $\Delta^{\delta}(x) \geq \Delta^{\varepsilon}(x)$ for all $x \in(0, \infty)$.

(2) $\Delta^{\varepsilon}(x) \leq x$ for all $x \in(0, \infty)$. 
(3) If $f \in \mathbb{F}_{\varepsilon}$ and $\mathrm{d}(x, y)>d$ then $\mathrm{d}(f(x), f(y)) \geq \Delta^{\varepsilon}(d)$.

Using the above we can easily see that if $\mathbf{d}^{p}\left(\mathrm{t}^{g}(a / A), \mathrm{t}^{g}(b / A)\right)<\Delta^{\varepsilon}(\varepsilon / 2)$ and $\mathbf{d}^{p}\left(\mathrm{t}^{g}(b / A), \mathrm{t}^{g}(c / A)\right)<\Delta^{\varepsilon}(\varepsilon / 2)$, then $\mathbf{d}^{p}\left(\mathrm{t}^{g}(a / A), \mathrm{t}^{g}(c / A)\right)<\varepsilon$.

Now for any set $A$ the sets

$$
D_{\varepsilon}^{p}=\left\{(p, q) \in S(A): \mathbf{d}^{p}(p, q) \leq \varepsilon\right\}
$$

form a base for a metrizable (diagonal) uniformity (see Chapter 9 of [13]). Thus, although $\mathbf{d}^{p}$ is not itself a metric, it makes sense to talk about Cauchysequences, limits and completeness with respect to $\mathbf{d}^{p}$.

To find limit types we use either goodness or completeness of type-spaces.

Lemma 4.4. Assume $A$ is good in $\mathfrak{M}$. Then if $\varepsilon>0$ and $\delta>0$ are as in the definition of goodness (Definition 2.20), and if $F_{n} \in \operatorname{Aut}_{\delta}(\mathfrak{M})$ for $n<\omega$ and for each $a \in A$ the sequence $\left(F_{n}(a)\right)$ converges then there is $F \in \operatorname{Aut}_{\varepsilon}(\mathfrak{M})$ such that for each $a \in A F(a)=\lim _{n \rightarrow \infty} F_{n}(a)$.

Proof. By the assumption weak $\delta$-embeddings $f: A \rightarrow \mathfrak{M}$ extend to $\varepsilon$ automorphisms of $\mathfrak{M}$. Hence it is enough to show that the function defined by $F(a)=\lim _{n \rightarrow \infty} F_{n}(a)$ for all $a \in A$ is a weak $\delta$-embedding. But this is exactly what the functions $F_{n}$ witness.

When using completeness of type-spaces we can actually find limit types over directed systems. Note that as soon as we have types actually extending each other and not just being $\mathbf{d}^{p}$-close, 0 -homogeneity gives these sorts of limit types:

Fact 4.5. Assume $(\mathcal{B}, \subseteq)$ is a directed system and $\left\{b_{B}: B \in \mathcal{B}\right\}$ are such that $\mathrm{t}^{g}\left(b_{B^{\prime}} / B\right)=\mathrm{t}^{g}\left(b_{B} / B\right)$ when $B \subset B^{\prime}, B, B^{\prime} \in \mathcal{B}$. Then there is some $b$ such that $\mathrm{t}^{g}(b / B)=\mathrm{t}^{g}\left(b_{B} / B\right)$ for all $B \in \mathcal{B}$.

Lemma 4.6. Assume $\mathbb{K}$ has complete type-spaces, $(\mathcal{B}, \subseteq)$ is a directed system of finite sets and $\left\{\mathrm{t}^{g}\left(b_{B}^{n} / B\right): n<\omega, B \in \mathcal{B}\right\}$ is $\mathbf{d}^{p}$-coherent in the following sense: for every $B \in \mathcal{B}$ and $\varepsilon>0$ there are $n_{0}<\omega$ and $B_{0} \in \mathcal{B}$ with $B_{0} \supseteq B$ such that for all $n \geq m \geq n_{0}$ and $B_{1}, B_{2} \in \mathcal{B}$ with $B_{2} \supseteq B_{1} \supseteq B_{0}$ $\mathbf{d}^{p}\left(\mathrm{t}^{g}\left(b_{B_{2}}^{n} / B_{1}\right), \mathrm{t}^{g}\left(b_{B_{1}}^{m} / B_{1}\right)\right) \leq \varepsilon$. Then there exists a limit type, i.e. there is some $b$ such that for every $B \in \mathcal{B}$ and $\varepsilon>0$ there is $n_{0}<\omega$ and $B_{0} \in \mathcal{B}$ with $B_{0} \supseteq B$ such that for all $n \geq n_{0}$ and $B_{1} \in \mathcal{B}$ with $B_{1} \supseteq B_{0}$ $\mathbf{d}^{p}\left(\mathrm{t}^{g}\left(b / B_{1}\right), \mathrm{t}^{g}\left(b_{B_{1}}^{n} / B_{1}\right)\right) \leq \varepsilon$. 
Proof. For each $B \in \mathcal{B}$ we find a $\mathbf{d}^{p}$-limit $b_{B}$ as follows: For every $m<\omega$ there are some $n_{m}<\omega$ and $B^{m} \supseteq B$ such that for all $n \geq n_{m}$ and $B^{\prime} \supseteq B^{m}$

$$
\mathbf{d}^{p}\left(\mathrm{t}^{g}\left(b_{B^{\prime}}^{n} / B^{m}\right), \mathrm{t}^{g}\left(b_{B^{m}}^{n_{m}} / B^{m}\right)\right) \leq \frac{1}{m}
$$

and we may choose $n_{m+1} \geq n_{m}$ and $B^{m+1} \supseteq B^{m}$ for all $m$. Then $\left(\mathrm{t}^{g}\left(b_{B^{m}}^{n_{m}} / B\right)\right)_{m<\omega}$ is $\mathbf{d}^{p}$-Cauchy and by completeness of type-spaces has a $\mathbf{d}^{p}$-limit $b_{B}$.

Now if $B \subset C \in \mathcal{B}$ then $\mathrm{t}^{g}\left(b_{C} / B\right)=\mathrm{t}^{g}\left(b_{B} / B\right)$ : For $\varepsilon>0$ choose $m$ large enough (and $>1 / \varepsilon$ ) such that

$$
\mathbf{d}^{p}\left(\mathrm{t}^{g}\left(b_{B} / B\right), \mathrm{t}^{g}\left(b_{B^{m}}^{n_{m}} / B\right)\right) \leq \varepsilon
$$

and similarly for $b_{C}$. Let $D \supset B^{m} \cup C^{m}$. Then by (4.1) for all large enough $n$

$$
\mathbf{d}^{p}\left(\mathrm{t}^{g}\left(b_{D}^{n} / B^{m}\right), \mathrm{t}^{g}\left(b_{B^{m}}^{n_{m}} / B^{m}\right)\right) \leq \frac{1}{m}<\varepsilon
$$

and similarly for $b_{C}$. Since this can be done for any $\varepsilon>0$, equality of the types $\mathrm{t}^{g}\left(b_{C} / B\right)$ and $\mathrm{t}^{g}\left(b_{B} / B\right)$ follows by perturbation. But then the claim follows by 0-homogeneity (Fact 4.5).

\section{SATURATiON}

Definition 5.1. We say that $A$ is $\omega$-d $\mathbf{d}^{p}$-saturated, if for all finite $A^{\prime} \subset A$, all $a \in \mathfrak{M}$ and all $\varepsilon>0$ there is $a^{\prime} \in A$ such that

$$
\mathbf{d}^{p}\left(\mathrm{t}^{g}\left(a / A^{\prime}\right), \mathrm{t}^{g}\left(a^{\prime} / A^{\prime}\right)\right) \leq \varepsilon
$$

(this is the same as demanding $\left.\mathbf{d}^{p}\left(\mathrm{t}^{g}\left(a A^{\prime} / \emptyset\right), \mathrm{t}^{g}\left(a^{\prime} A^{\prime} / \emptyset\right)\right) \leq \varepsilon\right)$.

Lemma 5.2. Assume $\mathbb{K}$ is $\omega$-d $\mathbf{d}^{p}$-stable. If $\mathcal{A}$ is $\omega$-d $\mathbf{d}^{p}$-saturated and $B \subset \mathcal{A}$ then there is an $\omega$-d $\mathbf{d}^{p}$-saturated $\mathcal{B} \preccurlyeq \mathcal{A}$ with $B \subseteq \mathcal{B}$ and $|\mathcal{B}|=|B|+\aleph_{0}$.

Proof. We build $\mathcal{B}$ as a chain of models $\overline{B_{n}}$, where for each $n<\omega$

- $\operatorname{card}\left(B_{n}\right) \leq|B|+\aleph_{0}$,

- $B_{n+1}$ realizes a $\mathbf{d}^{p}$-dense set of $S(A)$ for each finite $A \subset B_{n}$,

- $\overline{B_{n}} \preccurlyeq \mathcal{A}$.

We start by letting $B_{0}^{\prime}$ be a dense subset of $B$ of cardinality $|B|$ and then get $B_{0}$ by the assumption $\mathrm{LS}^{\mathrm{d}}=\aleph_{0}$. When $B_{n}$ has been defined we use $\omega$-d $\mathbf{d}^{p}$-stability and the $\mathrm{LS}^{\mathrm{d}}$ assumption to get $B_{n+1}$. Finally we let $\mathcal{B}=\overline{\bigcup_{n<\omega} B_{n}}$. Then clearly $\mathcal{B} \preccurlyeq \mathcal{A}$ and $|\mathcal{B}|=|B|+\aleph_{0}$. It is also easy to see that $\mathcal{B}$ is $\omega$-d $\mathbf{d}^{p}$-saturated, since parameters are allowed to move and we only need to realize types $\delta$-closely. 
Remark 5.3. It is easy to see how to adapt the proof to show that any $\omega$ $\mathbf{d}^{p}$-saturated set $A$ with a given subset $A^{\prime} \subset A$ contains an $\omega$-d $\mathbf{d}^{p}$-saturated set $B$ with $A^{\prime} \subseteq B$ and $|B|=\left|A^{\prime}\right|+\aleph_{0}$. Moreover, if $B$ is any $\omega$-d $\mathbf{d}^{p}$-saturated set, then $\bar{B}$ is also $\omega$-d $\mathbf{d}^{p}$-saturated.

Definition 5.4. We say that two models $\mathcal{A}$ and $\mathcal{B}$ are almost isomorphic if for each $\varepsilon>0$ there is an $\varepsilon$-isomorphism $f: \mathcal{A} \rightarrow \mathcal{B}$.

We will prove that $\omega$-d $\mathbf{d}^{p}$-saturated separable models are unique up to almost isomorphism. For this we need either goodness or the following continuity assumption for $\varepsilon$-isomorphisms:

Definition 5.5. We say that $\mathbb{K}$ is weakly $\mathbb{F}$-homogeneous if for every $\delta \geq 0$ if $f: \mathcal{A} \rightarrow \mathcal{B}$ is a weak $\delta$-embedding (see Definition 2.18) which is onto then $f \in \mathbb{F}_{\delta}$.

Remark 5.6. Note that $\mathbb{K}_{H}$ from section 3 is weakly $\mathbb{F}$-homogeneous.

Theorem 5.7. Assume $\mathcal{A}$ and $\mathcal{B}$ are separable and $\omega$-d $\mathbf{d}^{p}$-saturated. Then if either $\mathcal{A}$ and $\mathcal{B}$ are good or $\mathbb{K}$ is weakly $\mathbb{F}$-homogeneous, $\mathcal{A}$ and $\mathcal{B}$ are almost isomorphic.

Proof. First assume $\mathcal{A}$ and $\mathcal{B}$ are good. Fix $\varepsilon>0$. We will construct an $\varepsilon$-isomorphism between $\mathcal{A}$ and $\mathcal{B}$. So let $\delta>0$ be such that weak $\delta$-embeddings of both $\mathcal{A}$ and $\mathcal{B}$ extend to $\varepsilon$-embeddings, and choose for each $n<\omega, \delta_{n}>0$ such that $\delta_{n+1} \leq \delta_{n}$ for all $n$ and $\sum_{n<\omega} \delta_{n}<\delta$. Denote $\varepsilon_{n}=\sum_{i<n} \delta_{i}$. Let $A \subset \mathcal{A}$ and $B \subset \mathcal{B}$ be countable and dense and enumerate them $A=\left\{a_{n}: n<\omega\right\}, B=\left\{b_{n}: n<\omega\right\}$. We will define finite sets $A_{n} \subset \mathcal{A}, B_{n} \subset \mathcal{B}$ and mappings $f_{n}, g_{n}$ for $n<\omega$ such that

- $f_{n} \in \operatorname{Aut}_{\varepsilon_{2 n}}(\mathfrak{M})$ and maps $A_{n} \Delta^{2 \delta}\left(\delta_{2 n-1}\right)$-close to $\mathcal{B}$, i.e. there is $B_{n}^{\prime} \subset \mathcal{B}$ with $\left|B_{n}^{\prime}\right|=\left|A_{n}\right|$ such that $\mathrm{d}\left(f_{n}\left(A_{n}\right), B_{n}^{\prime}\right) \leq \Delta^{2 \delta}\left(\delta_{2 n-1}\right)$,

- $g_{n} \in \operatorname{Aut}_{\varepsilon_{2 n+1}}(\mathfrak{M})$ and maps $B_{n} \Delta^{2 \delta}\left(\delta_{2 n}\right)$-close to some $A_{n}^{\prime} \subset \mathcal{A}$,

- $A_{n+1}=A_{n} \cup\left\{a_{i}: i \leq n\right\} \cup A_{n}^{\prime}$,

- $B_{n+1}=B_{n} \cup\left\{b_{i}: i \leq n\right\} \cup B_{n+1}^{\prime}$.

We begin the construction by defining $A_{0}=B_{0}=\emptyset$ and $f_{0}=$ id. When $A_{n}, B_{n}$ and $f_{n}$ have been defined, consider the type $\mathrm{t}^{g}\left(f_{n}^{-1}\left(B_{n}\right) / A_{n}\right)$. Since $\mathcal{A}$ is $\omega$-d $\mathbf{d}^{p}$-saturated, there is some $A_{n}^{\prime} \subset \mathcal{A}$ such that

$$
\mathbf{d}^{p}\left(\mathrm{t}^{g}\left(f_{n}^{-1}\left(B_{n}\right) / A_{n}\right), \mathrm{t}^{g}\left(A_{n}^{\prime} / A_{n}\right)\right) \leq \Delta^{2 \delta}\left(\delta_{2 n}=.\right.
$$

Hence there is $g^{\prime} \in \operatorname{Aut}_{\delta_{2 n}}(\mathfrak{M})$ such that $\mathrm{d}\left(g^{\prime}\left(f_{n}^{-1}\left(B_{n}\right) A_{n}\right), A_{n}^{\prime} A_{n}\right) \leq$ $\Delta^{2 \delta}\left(\delta_{2 n}\right)$. Define $g_{n}=g^{\prime} \circ f_{n}^{-1}$. Then $g_{n} \in \operatorname{Aut}_{\varepsilon_{2 n+1}}(\mathfrak{M})$ and $g_{n}$ maps $B_{n} \Delta^{2 \delta}\left(\delta_{2 n}\right)$-close to $A_{n}^{\prime} \subset \mathcal{A}$. 
Similarly define $f_{n+1}$ and $B_{n+1}^{\prime}$ by considering $\mathrm{t}^{g}\left(g_{n}^{-1}\left(A_{n+1}\right) / B_{n}\right)$.

Now $g_{n} \circ f_{n}=g^{\prime}$ (as defined above) so $\mathrm{d}\left(g_{n} \circ f_{n}\left(A_{n}\right), A_{n}\right) \leq \Delta^{2 \delta}\left(\delta_{2 n}\right)$ and similarly we see that $\mathrm{d}\left(f_{n+1} \circ g_{n}\left(B_{n}\right), B_{n}\right) \leq \Delta^{2 \delta}\left(\delta_{2 n+1}\right)$. Hence

$$
\begin{aligned}
\mathrm{d}\left(f_{n+1}\left(A_{n}\right), f_{n}\left(A_{n}\right)\right) \leq & \mathrm{d}\left(f_{n+1}\left(A_{n}\right), f_{n+1} \circ g_{n} \circ f_{n}\left(A_{n}\right)\right) \\
& +\mathrm{d}\left(f_{n+1} \circ g_{n} \circ f_{n}\left(A_{n}\right), f_{n+1} \circ g_{n}\left(B_{n}^{\prime}\right)\right) \\
& +\mathrm{d}\left(f_{n+1} \circ g_{n}\left(B_{n}^{\prime}\right), B_{n}^{\prime}\right) \\
& +\mathrm{d}\left(B_{n}^{\prime}, f_{n}\left(A_{n}\right)\right) \\
\leq & 4 \delta_{2 n-1} .
\end{aligned}
$$

Hence we see that $\left(f_{n}\right)$ converges (pointwise) on $A$, and since the mappings are uniformly continuous with the same modulus of uniform continuity and $A$ is dense in $\mathcal{A}$, the sequence converges on $\mathcal{A}$. Since each $f_{n}$ is a $\delta$-automorphism, by Lemma 4.4 there is $F \in \operatorname{Aut}_{\varepsilon}(\mathfrak{M})$ such that $F(a)=\lim _{n \rightarrow \infty} f_{n}(a)$ for each $a \in \mathcal{A}$. Similarly we obtain $G \in \operatorname{Aut}_{\varepsilon}(\mathfrak{M})$ satisfying $G(b)=\lim _{n \rightarrow \infty} g_{n}(b)$ for each $b \in \mathcal{B}$.

To see that $F$ maps $\mathcal{A}$ into $\mathcal{B}$, note that for each $a \in \mathcal{A}, F(a)$ is the limit of a sequence of elements getting closer and closer to $\mathcal{B}$. Since $\mathcal{B}$ is metricly closed, we must have $F(a) \in \mathcal{B}$. Similarly we see that $G(b) \in \mathcal{A}$ for each $b \in \mathcal{B}$. To see that $F$ and $G$ are onto, it is enough to show that for all $a \in \mathcal{A}$ and $b \in \mathcal{B}, \mathrm{d}(G \circ F(a), a) \leq \delta$ and $\mathrm{d}(F \circ G(b), b) \leq \delta$ for any positive $\delta$. We prove the first claim since the latter is quite similar. So let $a \in \mathcal{A}$ and $\delta>0$ be given and choose $\delta^{\prime}<\Delta^{2 \varepsilon}(\delta / 7)$. Then we can find $a^{\prime} \in \bigcup_{n<\omega} A_{n}$, $b^{\prime} \in \bigcup_{n<\omega} B_{n}$ and $n<\omega$ such that

- $\mathrm{d}\left(a, a^{\prime}\right) \leq \delta^{\prime}$,

- $\mathrm{d}\left(F\left(a^{\prime}\right), f_{n}\left(a^{\prime}\right)\right) \leq \delta^{\prime}$,

- $\mathrm{d}\left(f_{n}\left(a^{\prime}\right), b^{\prime}\right) \leq \delta^{\prime}$,

- $\mathrm{d}\left(G\left(b^{\prime}\right), g_{n}\left(b^{\prime}\right)\right) \leq \delta^{\prime}$,

- $\mathrm{d}\left(g_{n} \circ f_{n}\left(a^{\prime}\right), a^{\prime}\right) \leq \delta^{\prime}$. 
Then

$$
\begin{aligned}
\mathrm{d}(G \circ F(a), a) \leq & \mathrm{d}\left(G \circ F(a), G \circ F\left(a^{\prime}\right)\right) \\
& +\mathrm{d}\left(G \circ F\left(a^{\prime}\right), G \circ f_{n}\left(a^{\prime}\right)\right) \\
& +\mathrm{d}\left(G \circ f_{n}\left(a^{\prime}\right), G\left(b^{\prime}\right)\right) \\
& +\mathrm{d}\left(G\left(b^{\prime}\right), g_{n}\left(b^{\prime}\right)\right) \\
& +\mathrm{d}\left(g_{n}\left(b^{\prime}\right), g_{n} \circ f_{n}\left(a^{\prime}\right)\right) \\
& +\mathrm{d}\left(g_{n} \circ f_{n}\left(a^{\prime}\right), a^{\prime}\right) \\
& +\mathrm{d}\left(a^{\prime}, a\right) \\
\leq & \delta .
\end{aligned}
$$

Now if instead of goodness we assume weak $\mathbb{F}$-homogeneity then just choose the $\delta_{n}$ such that $\sum_{n<\omega} \delta_{n}<\varepsilon$ and do the construction as above. Then $F$ and $G$ are defined as the limit mappings on $\mathcal{A}$ and $\mathcal{B}$ respectively, giving the required approximability by $\varepsilon$-functions. The onto-part is done as above and then weak $\mathbb{F}$-homogeneity will ensure that $F$ and $G$ are in $\mathbb{F}_{\varepsilon}$.

Remark 5.8. Note that if $A$ is almost isomorphic to an $\omega$-d $\mathbf{d}^{p}$-saturated set then $A$ is $\omega$-d $\mathbf{d}^{p}$-saturated.

\section{Splitting And independence}

Definition 6.1. Assume $A \subset B \subset \mathfrak{M}, A$ is finite and $a \in \mathfrak{M}$. We say that $\mathrm{t}^{g}(a / B) \varepsilon$-splits over $A$ if for all $\delta>0$ there are $b, c \in B$ such that $\mathbf{d}^{p}\left(\mathrm{t}^{g}(b / A), \mathrm{t}^{g}(c / A)\right) \leq \delta$ but $\mathbf{d}^{p}\left(\mathrm{t}^{g}(a b / A), \mathrm{t}^{g}(a c / A)\right)>\varepsilon$.

Splitting works nicely for $\omega$-d $\mathbf{d}^{p}$-stable classes $\mathbb{K}$, if we assume either goodness or completeness of type-spaces.

Theorem 6.2. If $\mathbb{K}$ is $\omega$-d $\mathbf{d}^{p}$-stable then for all $a \in \mathfrak{M}$, all separable good $A \subset \mathfrak{M}$ and $\varepsilon>0$ there is some finite $A^{\prime} \subset A$ such that $\mathrm{t}^{g}(a / A)$ does not E-split over $A^{\prime}$.

Proof. Let $A, a$ and $\varepsilon>0$ be given. If there is no finite $A^{\prime} \subset A$ such that $\mathrm{t}^{g}(a / A)$ does not $\varepsilon$-split over $A^{\prime}$ then for all finite $A_{n} \subset A$ and all $\delta_{n}>0$ there are $b_{n}, c_{n} \in A$ such that $\mathbf{d}^{p}\left(\mathrm{t}^{g}\left(b_{n} / A_{n}\right), \mathrm{t}^{g}\left(c_{n} / A_{n}\right)\right) \leq \delta_{n}$ but $\mathbf{d}^{p}\left(\mathrm{t}^{g}\left(a b_{n} / A_{n}\right), \mathrm{t}^{g}\left(a c_{n} / A_{n}\right)\right)>\varepsilon$. Let $\varepsilon^{\prime}<\varepsilon / 5$. Since $A$ is good, there is some $\delta>0$ such that weak $\delta$-embeddings of $A$ extend to $\varepsilon^{\prime}$-automorphisms of $\mathfrak{M}$ and we may assume $\delta \leq \Delta^{\varepsilon}\left(\varepsilon^{\prime}\right)$. Now we can define $A_{n}, b_{n}, c_{n}, f_{n}$ for $n<\omega$ such that 
- $A_{0}=\emptyset, A_{n} \subset A_{n+1} \subset A,\left|A_{n}\right|<\aleph_{0}$ and $\overline{\bigcup_{n<\omega} A_{n}} \supset A$,

- $\delta_{n}>0$ is such that $\sum_{n<\omega} \delta_{n}<\delta$,

- $\mathbf{d}^{p}\left(\mathrm{t}^{g}\left(b_{n} / A_{n}\right), \mathrm{t}^{g}\left(c_{n} / A_{n}\right)\right) \leq \Delta^{\delta}\left(\delta_{n}\right)$ and $\mathbf{d}^{p}\left(\mathrm{t}^{g}\left(a b_{n} / A_{n}\right), \mathrm{t}^{g}\left(a c_{n} / A_{n}\right)\right)>$ $\varepsilon$,

- $f_{n} \in \operatorname{Aut}_{\delta_{n}}(\mathfrak{M})$ and $\mathrm{d}\left(f_{n}\left(b_{n} A_{n}\right), c_{n} A_{n}\right) \leq \Delta^{\delta}\left(\delta_{n}\right)$,

- $b_{n}, c_{n} \in A_{n+1}$.

Now we can define $\delta$-automorphisms (of $\mathfrak{M}) F_{\eta\lceil n}$ for all $\eta \in{ }^{\omega} 2$ and $n<\omega$ as follows:

- $F_{\emptyset}=\mathrm{id}$,

- $F_{\eta\lceil n+1}= \begin{cases}F_{\eta\lceil n}, & \text { if } \eta(n)=0, \\ F_{\eta\lceil n} \circ f_{n}, & \text { if } \eta(n)=1 .\end{cases}$

Now $F_{\eta \mid n} \in \operatorname{Aut}_{\delta}(\mathfrak{M})$ for each $\eta \in{ }^{\omega} 2$ and $n<\omega$. Further

$$
\begin{aligned}
\mathrm{d}\left(F_{\eta\lceil n+1}\left(A_{n}\right), F_{\eta\lceil n}\left(A_{n}\right)\right) & \leq \mathrm{d}\left(F_{\eta\lceil n} \circ f_{n}\left(A_{n}\right), F_{\eta\lceil n}\left(A_{n}\right)\right) \\
& \leq \delta_{n} .
\end{aligned}
$$

Hence for each $\eta \in{ }^{\omega} 2,\left(F_{\eta\lceil n}\right)_{n<\omega}$ converges (pointwise) on $\bigcup_{n<\omega} A_{n}$ and since they have the same modulus of uniform continuity, on $A$. Thus by Lemma 4.4 there is $F_{\eta} \in \operatorname{Aut}_{\varepsilon^{\prime}}(\mathfrak{M})$ such that $F_{\eta}(c)=\lim _{n \rightarrow \infty} F_{\eta\lceil n}(c)$ for each $c \in A$.

Now let

$$
D=\bigcup\left\{F_{\eta \uparrow n}\left(A_{n}\right): \eta \in{ }^{\omega} 2, n<\omega\right\} .
$$

Then $D$ is countable. We wish to show that

$$
\mathbf{d}^{p}\left(\mathrm{t}^{g}\left(F_{\eta}(a) / D\right), \mathrm{t}^{g}\left(F_{\nu}(a) / D\right)\right)>\Delta^{\varepsilon}\left(\varepsilon^{\prime}\right)
$$

for all $\eta \neq \nu \in \omega^{\omega} 2$, hence arriving at a contradiction. For this we need the following:

Claim. $\mathbf{d}^{p}\left(\mathrm{t}^{g}\left(f_{n}\left(a b_{n} A_{n}\right) / \emptyset\right), \mathrm{t}^{g}\left(a c_{n} A_{n} / \emptyset\right)\right)>\varepsilon-\delta_{n}$.

Proof. Otherwise there would be some $f \in \operatorname{Aut}_{\varepsilon-\delta_{n}}(\mathfrak{M})$ with

$$
\mathrm{d}\left(f \circ f_{n}\left(a b_{n} A_{n}\right), a c_{n} A_{n}\right) \leq \varepsilon-\delta_{n},
$$

but then $f \circ f_{n} \in \operatorname{Aut}_{\varepsilon}(\mathfrak{M})$ and $\mathbf{d}^{p}\left(\mathrm{t}^{g}\left(a b_{n} / A_{n}\right), \mathrm{t}^{g}\left(a c_{n} / A_{n}\right)\right) \leq \varepsilon$, a contradiction.

Now let $\eta, \nu \in{ }^{\omega} 2, \eta \neq \nu$ and $n=\min \{n: \eta(n) \neq \nu(n)\}$. We may assume $\eta(n)=0$ and then use the following:

- for any $\zeta \in{ }^{\omega} 2, \mathrm{~d}\left(F_{\zeta}\left(A_{n+1}\right), F_{\zeta\lceil n+1}\left(A_{n+1}\right)\right) \leq \delta \leq \Delta^{\varepsilon}\left(\varepsilon^{\prime}\right)$,

- $\mathrm{d}\left(F_{\nu \uparrow n+1}\left(b_{n} A_{n}\right), F_{\eta\lceil n+1}\left(c_{n} A_{n}\right)\right)=\mathrm{d}\left(F_{\nu \mid n} \circ f_{n}\left(b_{n} A_{n}\right), F_{\nu \mid n}\left(c_{n} A_{n}\right)\right) \leq$ $\delta_{n}$. 
Then we must have

$$
\mathbf{d}^{p}\left(\mathrm{t}^{g}\left(F_{\nu}(a) / F_{\eta\lceil n+1}\left(A_{n+1}\right)\right), \mathrm{t}^{g}\left(F_{\eta}(a) / F_{\eta\lceil n+1}\left(A_{n+1}\right)\right)\right)>\Delta^{\varepsilon}\left(\varepsilon^{\prime}\right)
$$

since otherwise we can find $g \in$ Aut $_{\varepsilon^{\prime}}$ with

$$
\mathrm{d}\left(g\left(F_{\nu}(a) F_{\eta\lceil n+1}\left(A_{n+1}\right)\right), F_{\eta}(a) F_{\eta\lceil n+1}\left(A_{n+1}\right)\right) \leq \Delta^{\varepsilon}\left(\varepsilon^{\prime}\right) .
$$

and then

$$
F_{\eta}^{-1} \circ g \circ F_{\nu} \circ f_{n}^{-1} \in \mathrm{Aut}_{4 \varepsilon^{\prime}}
$$

and

$$
\begin{aligned}
& \mathrm{d}\left(F_{\eta}^{-1} \circ g \circ F_{\nu} \circ f_{n}^{-1}\left(f_{n}\left(a b_{n} A_{n}\right)\right), a c_{n} A_{n}\right) \\
&=\mathrm{d}\left(F_{\eta}^{-1} \circ g \circ F_{\nu}\left(a b_{n} A_{n}\right), a c_{n} A_{n}\right) \\
& \leq \quad \mathrm{d}\left(F_{\eta}^{-1} \circ g \circ F_{\nu}\left(a b_{n} A_{n}\right), F_{\eta}^{-1} \circ g\left(F_{\nu}(a) F_{\nu\lceil n+1}\left(b_{n} A_{n}\right)\right)\right) \\
& \quad+\mathrm{d}\left(F_{\eta}^{-1} \circ g\left(F_{\nu}(a) F_{\nu\lceil n+1}\left(b_{n} A_{n}\right)\right), F_{\eta}^{-1} \circ g\left(F_{\nu}(a) F_{\eta\lceil n+1}\left(c_{n} A_{n}\right)\right)\right) \\
& \quad+\mathrm{d}\left(F_{\eta}^{-1} \circ g\left(F_{\nu}(a) F_{\eta\lceil n+1}\left(c_{n} A_{n}\right)\right), F_{\eta}^{-1}\left(F_{\eta}(a) F_{\eta\lceil n+1}\left(c_{n} A_{n}\right)\right)\right) \\
& \quad+\mathrm{d}\left(F_{\eta}^{-1}\left(F_{\eta}(a) F_{\eta\lceil n+1}\left(c_{n} A_{n}\right)\right), a c_{n} A_{n}\right) \\
& \leq 4 \varepsilon^{\prime},
\end{aligned}
$$

showing that $\mathbf{d}^{p}\left(\mathrm{t}^{g}\left(f_{n}\left(a b_{n} A_{n}\right) / \emptyset\right), \mathrm{t}^{g}\left(a c_{n} A_{n} / \emptyset\right)\right) \leq 4 \varepsilon^{\prime}<\varepsilon-\delta_{n}$, contradicting the claim (the other direction needed by the symmetry of $\mathbf{d}^{p}$ is proved similarly).

Theorem 6.3. If $\mathbb{K}$ is $\omega$-d $\mathbf{d}^{p}$-stable and all models are good (i.e. $(\mathcal{A}, \mathcal{A})$ is good for all $\mathcal{A} \in \mathbb{K})$ then for all $a \in \mathfrak{M}, \mathcal{A} \preccurlyeq \mathfrak{M}$ and $\varepsilon>0$ there is some finite $A^{\prime} \subset \mathcal{A}$ such that $\mathrm{t}^{g}(a / \mathcal{A})$ does not $\varepsilon$-split over $A^{\prime}$.

Proof. Assume towards a contradiction that $\mathcal{B}, a$ and $\varepsilon>0$ are such that $\mathrm{t}^{g}(a / \mathcal{B}) \varepsilon$-splits over every finite subset of $\mathcal{B}$. Then we may construct a separable $\mathcal{A} \in \mathcal{B}$ as the closure of a countable union of at most countable sets as follows:

First let $A_{0} \subset \mathcal{B}$ be any finite set. When $A_{n}$ has been defined and is at most countable, by assumption for any finite $A^{\prime} \subset A_{n}$ and any rational $r>0$ there are $b_{r}^{\prime}, c_{r}^{\prime} \in \mathcal{B}$ such that $\mathbf{d}^{p}\left(\mathrm{t}^{g}\left(b_{r}^{\prime} / A^{\prime}\right), \mathrm{t}^{g}\left(c_{r}^{\prime} / A^{\prime}\right)\right) \leq r$ but $\mathbf{d}^{p}\left(\mathrm{t}^{g}\left(a b_{r}^{\prime} / A^{\prime}\right), \mathrm{t}^{g}\left(a c_{r}^{\prime} / A^{\prime}\right)\right)>\varepsilon$. Then let $A_{n+1}$ be a countable set containing $A_{n} \cup\left\{b_{r}^{\prime}, c_{r}^{\prime}: r \in \mathbb{Q}, r>0, A^{\prime} \subset A_{n}\right.$ finite $\}$ and such that $\overline{A_{n+1}} \preccurlyeq \mathcal{B}$. In the end let

$$
\mathcal{A}=\overline{\bigcup_{n<\omega} A_{n}}=\overline{\bigcup_{n<\omega} \overline{A_{n}}} \preccurlyeq \mathcal{B}
$$

Then $\mathcal{A}$ is separable but $\mathrm{t}^{g}(a / \mathcal{A}) \varepsilon^{\prime}$-splits over all finite $A \subset \mathcal{A}$ when $\varepsilon^{\prime} \leq \varepsilon / 2$, contradicting Theorem 6.2. Namely let $A \subset \mathcal{A}$ be finite and $\delta>0$ 
and we may assume $\delta \leq \varepsilon^{\prime}$. Now choose some rational $r \leq \delta / 2$ and a finite $A^{\prime} \subset \bigcup_{n<\omega} A_{n}$ with $\mathrm{d}\left(A, A^{\prime}\right) \leq \Delta^{\varepsilon^{\prime}}(\delta / 4)$. Then $A^{\prime} \in A_{n}$ for some $n<\omega$ and there are $b_{r}^{\prime}, c_{r}^{\prime} \in \mathcal{A}$ such that

$$
\mathbf{d}^{p}\left(\mathrm{t}^{g}\left(b_{r}^{\prime} / A^{\prime}\right), \mathrm{t}^{g}\left(c_{r}^{\prime} / A^{\prime}\right)\right) \leq r
$$

but

$$
\mathbf{d}^{p}\left(\mathrm{t}^{g}\left(a b_{r}^{\prime} / A^{\prime}\right), \mathrm{t}^{g}\left(a c_{r}^{\prime} / A^{\prime}\right)\right)>\varepsilon .
$$

Now if $f$ is an $r$-function witnessing (6.1), we have

$$
\mathrm{d}(f(A), A) \leq \mathrm{d}\left(f(A), f\left(A^{\prime}\right)\right)+\mathrm{d}\left(f\left(A^{\prime}\right), A^{\prime}\right)+\mathrm{d}\left(A^{\prime}, A\right) \leq \delta .
$$

Hence $\mathbf{d}^{p}\left(\mathrm{t}^{g}\left(b_{r}^{\prime} / A\right), \mathrm{t}^{g}\left(c_{r}^{\prime} / A\right)\right) \leq \delta$. But $\mathbf{d}^{p}\left(\mathrm{t}^{g}\left(a b_{r}^{\prime} / A\right), \mathrm{t}^{g}\left(a c_{r}^{\prime} / A\right)\right)$ must be at least $\varepsilon^{\prime}$ since if there were an $\varepsilon^{\prime}$-function $g$ witnessing the contrary, we would have $\mathrm{d}\left(g\left(a b_{r}^{\prime}\right), a c_{r}^{\prime}\right) \leq \varepsilon^{\prime}$ and $\mathrm{d}\left(g\left(A^{\prime}\right), A^{\prime}\right) \leq \delta / 4+\varepsilon^{\prime}+\delta / 4<2 \varepsilon^{\prime} \leq \varepsilon$, contradicting $(6.2)$

Theorem 6.4. If $\mathbb{K}$ is $\omega$ - $\mathbf{d}^{p}$-stable and has complete type-spaces then for all $a \in \mathfrak{M}, A \subset \mathfrak{M}$ and $\varepsilon>0$ there is some finite $A^{\prime} \subset A$ such that $\mathrm{t}^{g}(a / A)$ does not $\varepsilon$-split over $A^{\prime}$.

Proof. This is proved by a tree construction just as in Theorem 6.2, but here we may directly choose $\delta<\Delta^{\varepsilon}\left(\varepsilon / 7\right.$ ) (and forget about $\varepsilon^{\prime}$ ) and we just need $A_{n}$ to form an increasing sequence of finite subsets of $A$, without any demands on the union. Then the automorphisms $F_{\eta \uparrow n}$ are defined as before. Now for each $\eta \in{ }^{\omega} 2,\left\{\mathrm{t}^{g}\left(F_{\eta\lceil n}(a) / F_{\eta\lceil n}\left(A_{n}\right)\right): n<\omega\right\}$ forms a coherent system in the sense that for each $\varepsilon>0$ there is some $n_{0}<\omega$ such that for $n>m>n_{0} \mathbf{d}^{p}\left(\mathrm{t}^{g}\left(F_{\eta\lceil n}(a) / F_{\eta\lceil m}\left(A_{m}\right)\right), \mathrm{t}^{g}\left(F_{\eta\lceil m}(a) / F_{\eta\lceil m}\left(A_{m}\right)\right)\right)<\varepsilon$. Hence by Lemma 4.6 there exists a limit type of the sequence

$$
\left(\mathrm{t}^{g}\left(F_{\eta\lceil n}(a) / F_{\eta\lceil n}\left(A_{n}\right)\right)\right)_{n<\omega}
$$

realized by some $a_{\eta}$.

Now let $\eta \neq \nu$ with $n=\min \{n: \eta(n) \neq \nu(n)\}$ and again we may assume $\eta(n)=0$. By choosing $N$ large enough we can find $g_{\eta}, g_{\nu} \in$ Aut $_{\delta}$ such that

$$
\mathrm{d}\left(g_{\nu}\left(F_{\nu\lceil N}\left(a A_{n+1}\right)\right), a_{\nu} F_{\nu \uparrow N}\left(A_{n+1}\right)\right)<\delta
$$

and

$$
\mathrm{d}\left(g_{\eta}\left(a_{\eta} F_{\eta \uparrow N}\left(A_{n+1}\right), F_{\eta \uparrow N}\left(a A_{n+1}\right)\right)\right)<\delta .
$$

Then we can easily calculate that

- for any $\zeta \in{ }^{\omega} 2$ and $N>n, \mathrm{~d}\left(F_{\zeta \uparrow N}\left(A_{n+1}\right), F_{\zeta\lceil n+1}\left(A_{n+1}\right)\right)<\delta$,

- $\mathrm{d}\left(F_{\nu\lceil n+1}\left(b_{n} A_{n}\right), F_{\eta\lceil n+1}\left(c_{n} A_{n}\right)\right)<\delta_{n}$. 
As in Therem 6.2 we can prove that

$$
\mathbf{d}^{p}\left(\mathrm{t}^{g}\left(f_{n}\left(a b_{n} A_{n}\right) / \emptyset\right), \mathrm{t}^{g}\left(a c_{n} A_{n} / \emptyset\right)\right)>\varepsilon-\delta_{n} .
$$

So, similarly as in Theorem 6.2, we must have

$$
\mathbf{d}^{p}\left(\mathrm{t}^{g}\left(a_{\nu} / F_{\eta\lceil n+1}\left(A_{n+1}\right), \mathrm{t}^{g}\left(a_{\eta} / F_{\eta\lceil n+1}\left(A_{n+1}\right)\right)\right)>\delta,\right.
$$

since otherwise we can find $g \in \operatorname{Aut}_{\delta}$ with $\mathrm{d}\left(g\left(a_{\nu} / F_{\eta \mid n+1}\left(A_{n+1}\right)\right), a_{\eta} / F_{\eta\lceil n+1}\left(A_{n+1}\right)\right)<$ $\delta$ and then

$$
F_{\eta\lceil N}^{-1} \circ g_{\eta} \circ g \circ g_{\nu} \circ F_{\nu \nmid N} \circ f_{n}^{-1} \in \mathrm{Aut}_{6 \delta}
$$

and

$$
\begin{aligned}
& \mathrm{d}\left(F_{\eta\lceil N}^{-1} \circ g_{\eta} \circ g \circ g_{\nu} \circ F_{\nu \uparrow N} \circ f_{n}^{-1}\left(f_{n}\left(a b_{n} A_{n}\right)\right), a c_{n} A_{n}\right) \\
& =\mathrm{d}\left(F_{\eta\lceil N}^{-1} \circ g_{\eta} \circ g \circ g_{\nu} \circ F_{\nu \uparrow N}\left(a b_{n} A_{n}\right), a c_{n} A_{n}\right) \\
& \leq \mathrm{d}\left(F_{\eta \uparrow N}^{-1} \circ g_{\eta} \circ g \circ g_{\nu}\left(F_{\nu \uparrow N}\left(a b_{n} A_{n}\right)\right), F_{\eta \uparrow N}^{-1} \circ g_{\eta} \circ g\left(a_{\nu} F_{\nu \uparrow N}\left(b_{n} A_{n}\right)\right)\right) \\
& +\mathrm{d}\left(F_{\eta \uparrow N}^{-1} \circ g_{\eta} \circ g\left(a_{\nu} F_{\nu \uparrow N}\left(b_{n} A_{n}\right)\right), F_{\eta \uparrow N}^{-1} \circ g_{\eta} \circ g\left(a_{\nu} F_{\nu\lceil n+1}\left(b_{n} A_{n}\right)\right)\right) \\
& +\mathrm{d}\left(F_{\eta\lceil N}^{-1} \circ g_{\eta} \circ g\left(a_{\nu} F_{\nu\lceil n+1}\left(b_{n} A_{n}\right)\right), F_{\eta\lceil N}^{-1} \circ g_{\eta} \circ g\left(a_{\nu} F_{\eta\lceil n+1}\left(c_{n} A_{n}\right)\right)\right) \\
& +\mathrm{d}\left(F_{\eta\lceil N}^{-1} \circ g_{\eta} \circ g\left(a_{\nu} F_{\eta\lceil n+1}\left(c_{n} A_{n}\right)\right), F_{\eta\lceil N}^{-1} \circ g_{\eta}\left(a_{\eta} F_{\eta\lceil n+1}\left(c_{n} A_{n}\right)\right)\right) \\
& +\mathrm{d}\left(F_{\eta\lceil N}^{-1} \circ g_{\eta}\left(a_{\eta} F_{\eta\lceil n+1}\left(c_{n} A_{n}\right)\right), F_{\eta\lceil N}^{-1} \circ g_{\eta}\left(a_{\eta} F_{\eta\lceil N}\left(c_{n} A_{n}\right)\right)\right) \\
& +\mathrm{d}\left(F_{\eta \uparrow N}^{-1} \circ g_{\eta}\left(a_{\eta} F_{\eta \uparrow N}\left(c_{n} A_{n}\right)\right), a c_{n} A_{n}\right) \\
& \leq 6 \varepsilon / 7<\varepsilon-\delta_{n},
\end{aligned}
$$

contradicting (6.3). Now define $D=\bigcup\left\{F_{\eta\lceil n}\left(A_{n}\right): \eta \in{ }^{\omega} 2, n<\omega\right\}$. Then $D$ is countable and (6.4) gives a contradiction with the assumption of $\omega$-d $\mathbf{d}^{p_{-}}$ stability.

Based on $\varepsilon$-splitting we can define a notion of independence.

Definition 6.5. We write $a \downarrow_{A}^{\varepsilon} B$ if there is some finite $A^{\prime} \subseteq A$ such that $\mathrm{t}^{g}(a / A \cup B)$ does not $\varepsilon$-split over $A^{\prime}$. We then define $a \downarrow_{A}^{s} B$ if for all $\varepsilon>0$, $a \downarrow_{A}^{\varepsilon} B$.

We now set out to prove that this notion of independence satisfies the usual axioms for an independence notion. The axiom of finite character is replaced by countable character, as is to be expected in a metric setting.

Theorem 6.6. If $\mathbb{K}$ is $\omega$-d $\mathbf{d}^{p}$-stable and has complete type spaces then independence satisfies the following axioms:

(1) Isomorphism invariance If $a \downarrow_{A}^{s} B$ and $f$ is a 0-automorphism of $\mathfrak{M}$ then $f(a) \downarrow_{f(A)}^{s} f(B)$.

(2) Monotonicity If $A \subseteq B \subseteq C \subseteq D$ and $a \downarrow_{A}^{s} D$ then $a \downarrow_{B}^{s} C$. 
(3) Countable character of non-freeness If $A$ is $\omega$-d $\mathbf{d}^{p}$-saturated and a $\chi_{A}^{s} B$ then there is a countable $A^{\prime} \subseteq A$ and a finite $B^{\prime} \subseteq B$ such that if $\mathrm{t}^{g}\left(b / A^{\prime} B^{\prime}\right)=\mathrm{t}^{g}\left(a / A^{\prime} B^{\prime}\right)$ then $b \quad \chi_{A}^{s} B^{\prime}$.

(4) Local character $a \downarrow_{A}^{s} A$ for all $a$ and $A$, i.e. for every $a, A$ and $\varepsilon>0$ there is some finite $E \subseteq A$ such that a $\downarrow_{E}^{\varepsilon} A$.

(5) Extension If a $\downarrow_{A}^{s} B, B$ is $\omega$-d $\mathbf{d}^{p}$-saturated and $A \subseteq B \subseteq C$ then there is $b$ with $\mathrm{t}^{g}(b / B)=\mathrm{t}^{g}(a / B)$ satisfying $b \downarrow_{A}^{s} C$.

(6) Stationarity If $A$ is $\omega$-d $\mathbf{d}^{p}$-saturated, $A \subseteq B$, $\mathrm{t}^{g}(a / A)=\mathrm{t}^{g}(b / A)$, $a \downarrow_{A}^{s} B$ and $b \downarrow_{A}^{s} B$, then $\mathrm{t}^{g}(a / B)=\mathrm{t}^{g}(b / B)$.

(7) Transitivity If $A \subseteq B \subseteq C, B$ is $\omega$-d $\mathbf{d}^{p}$-saturated, $a \downarrow_{A}^{s} B$ and $a \downarrow_{B}^{s} C$ then $a \downarrow_{A}^{s} C$.

(8) Symmetry If $A$ is $\omega-\mathbf{d}^{p}$-saturated then $a \downarrow_{A}^{s} b$ if and only if $b \downarrow_{A}^{s}$ a.

(9) Reflexivity If $A$ is $\omega$-d $\mathbf{d}^{p}$-saturated and metricly closed and $a \notin A$ then a $\not_{A}^{s}$ a.

Remark 6.7. We will see that the assumption of complete type-spaces is used only in the proofs of local character and extension and then the extension property is used to prove transitivity and symmetry. Hence, we may exchange the assumption of complete type-spaces for the assumptions that models are good and extension holds. Then all the axioms hold with the minor change that local character is true only when $A$ is a model.

Proof. (of Theorem 6.6) By the definition it is clear that independence is preserved under 0-automorphisms and is monotone. Local character is Theorem 6.4.

Countable character of non-freeness: Let $A^{\prime} \subset A$ be countable such that $\overline{A^{\prime}}$ is $\omega$-d $\mathbf{d}^{p}$-saturated and $a \downarrow_{A^{\prime}}^{s} A$. Let $a^{\prime}$ be such that $\mathrm{t}^{g}\left(a^{\prime} / A\right)=$ $\mathrm{t}^{g}(a / A)$ and $a^{\prime} \downarrow_{A}^{s} B$. Then let $B^{\prime} \subseteq B$ be such that $\mathrm{t}^{g}\left(a / B^{\prime}\right) \neq \mathrm{t}^{g}\left(a^{\prime} / B^{\prime}\right)$. The claim follows by stationarity which is proved below.

Extension: Let, for each $n<\omega, A_{n} \subset A$ be finite and such that $\mathrm{t}^{g}(a / B)$ does not $\frac{1}{n+1}$-split over $A_{n}$. Then for each finite $c \in C$, by $\omega$-d $\mathbf{d}^{p}$-saturation of $B$, let $c_{n}^{c} \in B$ be such that $\mathbf{d}^{p}\left(\mathrm{t}^{g}\left(c_{n}^{c} / A_{n}\right), \mathrm{t}^{g}\left(c / A_{n}\right)\right) \leq \frac{1}{n+1}$ and let $f_{n}^{c}$ be a $\frac{1}{n+1}$-automorphism of $\mathfrak{M}$ witnessing this. Then define $a_{n}^{c}=f_{n}^{c}(a)$. We claim that $\left\{\mathrm{t}^{g}\left(a_{n}^{c} / A_{n} c\right): n<\omega, c \in C\right.$ finite $\}$ forms a $\mathbf{d}^{p}$-coherent system in the sense of Lemma 4.6. Namely, if $c \in C$ and $\varepsilon>0$ are given, then choose $n_{1}$ large enough such that $\frac{1}{n_{1}}<\Delta^{\varepsilon}(\varepsilon / 3)$. Now $\mathrm{t}^{g}(a / B)$ does not $\frac{1}{n_{1}}$-split over $A_{n_{1}}$ and there is some $\delta>0$ witnessing this. Choose $n_{0} \geq n_{1}$ such that $\frac{2}{n_{0}} \leq \Delta^{\varepsilon}(\delta)$ and let $B_{0}=A_{n_{1}} \cup c$. Then if $n \geq m \geq n_{0}$, for any finite $d, e \in C$, we need to show that $\mathbf{d}^{p}\left(\mathrm{t}^{g}\left(a_{n}^{c d e} / A_{n_{1}} c d\right), \mathrm{t}^{g}\left(a_{m}^{c d} / A_{n_{1}} c d\right)\right) \leq \varepsilon$. But 
now

and

$$
\mathbf{d}^{p}\left(\mathrm{t}^{g}\left(a_{n}^{c d e} c d / A_{n_{1}}\right), \mathrm{t}^{g}\left(a,(c d)_{n}^{c d e} / A_{n_{1}}\right)\right) \leq \frac{1}{n_{1}}
$$

Further

$$
\mathbf{d}^{p}\left(\mathrm{t}^{g}\left(a_{m}^{c d} c d / A_{n_{1}}\right), \mathrm{t}^{g}\left(a,(c d)_{m}^{c d} / A_{n_{1}}\right)\right) \leq \frac{1}{n_{1}}
$$

$$
\mathbf{d}^{p}\left(\mathrm{t}^{g}\left((c d)_{n}^{c d e} / A_{n_{1}}\right), \mathrm{t}^{g}\left((c d) c d_{m} / A_{n_{1}}\right)\right) \leq \delta
$$

and $(c d)_{n}^{c d e},(c d)_{m}^{c d} \in B$, so the claim follows by non- $\frac{1}{n_{1}}$-splitting. Hence by Lemma 4.6 there is some $b$ realizing the limit type of all $a_{n}^{c}$ 's, i.e. for all finite $c \in C$ and $\varepsilon>0$ there is $n_{0}<\omega$ and $c_{0} \in C$ such that for all $n>n_{0}$ and $d \in C$ with $c_{0} \subseteq d, \mathbf{d}^{p}\left(\mathrm{t}^{g}\left(b / A_{n} d\right), \mathrm{t}^{g}\left(a_{n}^{d} / A_{n} d\right)\right) \leq \varepsilon$.

Next we claim $\mathrm{t}^{g}(b / B)=\mathrm{t}^{g}(a / B)$. For this it suffices to prove that $\mathbf{d}^{p}\left(\mathrm{t}^{g}(b c / \emptyset), \mathrm{t}^{g}(a c / \emptyset)\right) \leq \varepsilon$ for any $\varepsilon>0$ and finite $c \in B$. So let $\varepsilon>0$ and $c \in B$ be given and let $n$ be large enough so that $\mathrm{t}^{g}(a / B)$ does not $\varepsilon / 3$-split over $A_{n}$. Then let $\delta>0$ witness this and be at most $\Delta^{\varepsilon}(\varepsilon / 3)$. Then choosing $m$ large enough, we can ensure

- $\mathbf{d}^{p}\left(\mathrm{t}^{g}\left(b c / A_{n}\right), \mathrm{t}^{g}\left(a_{m}^{c} c / A_{n}\right)\right) \leq \delta$ and

- $\mathbf{d}^{p}\left(\mathrm{t}^{g}\left(a_{m}^{c} c / A_{n}\right), \mathrm{t}^{g}\left(a c_{m}^{c} / A_{n}\right)\right) \leq \delta$.

Then by non-splitting we get $\mathbf{d}^{p}\left(\mathrm{t}^{g}\left(a c_{m}^{c} / A_{n}\right), \mathrm{t}^{g}\left(a c / A_{n}\right)\right) \leq \varepsilon / 3$. Combining these we get $\mathbf{d}^{p}\left(\mathrm{t}^{g}\left(b c / A_{n}\right), \mathrm{t}^{g}\left(a c / A_{n}\right)\right) \leq \varepsilon$.

Finally we need to show that $b \downarrow_{A}^{s} C$. We actually claim that $b \downarrow_{A_{n}}^{\frac{1}{n+1}} C$. But this is easy to see, since if for every $\delta>0$ there were $c, d \in C$ witnessing $\frac{1}{n+1}$ splitting, then for some $m$ large enough $\mathbf{d}^{p}\left(\mathrm{t}^{g}\left(c_{m}^{c} / A_{n}\right), \mathrm{t}^{g}\left(d_{m}^{d} / A_{n}\right)\right)$ would be small enough to contradict the assumption $a \downarrow_{A_{n}}^{\frac{1}{n+1}} B$, since

$$
\mathbf{d}^{p}\left(\mathrm{t}^{g}\left(b c / A_{n}\right), \mathrm{t}^{g}\left(a_{m}^{c} c / A_{n}\right)\right)
$$

can be made arbitrarily small with a large enough $m$ and

$$
\mathbf{d}^{p}\left(\mathrm{t}^{g}\left(a_{m}^{c} c / A_{n}\right), \mathrm{t}^{g}\left(a c_{m}^{c} / A_{n}\right)\right) \leq \frac{1}{m}
$$

for $m \geq n$ (and similarly for $d$ ).

Stationarity: To show that $\mathrm{t}^{g}(a / B)=\mathrm{t}^{g}(b / B)$, we need to show that for all finite $c \in B \mathrm{t}^{g}(a c / \emptyset)=\mathrm{t}^{g}(b c / \emptyset)$. By perturbation, it is enough to show that for all finite $c \in B$ and all $\varepsilon>0, \mathbf{d}^{p}\left(\mathrm{t}^{g}(a c / \emptyset), \mathrm{t}^{g}(b c / \emptyset)\right) \leq \varepsilon$. So fix some finite $c \in B$ and $\varepsilon>0$. By the assumption there is some finite $A^{\prime} \subset A$ such that $\mathrm{t}^{g}(a / B)$ and $\mathrm{t}^{g}(b / B)$ do not $\Delta^{\varepsilon}(\varepsilon / 2)$-split over $A^{\prime}$ and there is some $\delta>0$ witnessing this. Since $A$ is $\omega$-d $\mathbf{d}^{p}$-saturated, there is $c^{\prime} \in A$ such that $\mathbf{d}^{p}\left(\mathrm{t}^{g}\left(c^{\prime} / A^{\prime}\right), \mathrm{t}^{g}\left(c / A^{\prime}\right)\right) \leq \delta$. Then

$$
\text { - } \mathbf{d}^{p}\left(\mathrm{t}^{g}(a c / \emptyset), \mathrm{t}^{g}\left(a c^{\prime} / \emptyset\right)\right) \leq \Delta^{\varepsilon}(\varepsilon / 2) \text {, }
$$


- $\mathbf{d}^{p}\left(\mathrm{t}^{g}\left(a c^{\prime} / \emptyset\right), \operatorname{tg}\left(b c^{\prime} / \emptyset\right)\right)=0$ and

- $\mathbf{d}^{p}\left(\mathrm{t}^{g}\left(b c^{\prime} / \emptyset\right), \mathrm{t}^{g}(b c / \emptyset)\right) \leq \Delta^{\varepsilon}(\varepsilon / 2)$.

Combining we get

$$
\mathbf{d}^{p}\left(\mathrm{t}^{g}(a c / \emptyset), \mathrm{t}^{g}(b c / \emptyset)\right) \leq \varepsilon .
$$

Transitivity: By the extension property let $b$ be such that $\mathrm{t}^{g}(b / B)=$ $\mathrm{t}^{g}(a / B)$ and $b \downarrow_{A}^{s} C$. Then $a \downarrow_{B}^{s} C$ and $b \downarrow_{B}^{s} C$ so by stationarity $\mathrm{t}^{g}(a / C)=$ $\mathrm{t}^{g}(b / C)$, hence $a \downarrow_{A}^{s} C$.

Symmetry: The proof is postponed until Lemma 6.13.

Reflexivity: Let $d$ be the distance of $a$ to $A$, i.e. $d=\inf \left\{\mathrm{d}\left(a, a^{\prime}\right): a^{\prime} \in A\right\}$. Since $A$ is a closed subset of $\mathfrak{M}, d>0$. Now t ${ }^{g}(a / A a) \Delta^{d}(d) / 3$-splits over any finite $A^{\prime} \subset A$. Namely let $A^{\prime} \subset A$ be finite and $\delta>0$. By $\omega$-d $\mathbf{d}^{p_{-}}$ saturation, let $a^{\prime} \in A$ be such that $\mathbf{d}^{p}\left(\mathrm{t}^{g}\left(a^{\prime} / A^{\prime}\right), \mathrm{t}^{g}\left(a / A^{\prime}\right)\right) \leq \delta$. Then if $f \in \mathrm{Aut}_{\varepsilon}$ for some $\varepsilon \leq d, \mathrm{~d}\left(f\left(a a^{\prime} A^{\prime}\right), a a^{\prime} A^{\prime}\right) \geq \Delta^{\varepsilon}(d) / 2 \geq \Delta^{d}(d) / 2$ and thus $\mathbf{d}^{p}\left(\mathrm{t}^{g}\left(a a^{\prime} / A^{\prime}\right), \mathrm{t}^{g}\left(a a / A^{\prime}\right)\right)>\Delta^{d}(d) / 3$.

Although we have a weaker stability notion than in [9], the $\omega$-stability notion at hand implies discrete $\lambda$-stability for all $\lambda$ with $\lambda=\lambda^{\aleph_{0}}$.

Lemma 6.8. If $\mathbb{K}$ is $\boldsymbol{\omega}$-d $\mathbf{d}^{p}$-stable and either has complete type-spaces or good models (i.e. $(\mathcal{A}, \mathcal{A})$ is good for all $\mathcal{A} \in \mathbb{K}$ ) then for all $\lambda=\lambda^{\aleph_{0}} \mathbb{K}$ is $\lambda$-stable in the discrete sense i.e. the number of types over a set of density character $\lambda$ is $\lambda$ (which then also is the cardinality of the set).

Proof. Let $|B| \leq \lambda$ and let $a, b \in \mathfrak{M}$. By Lemma 5.2 we may assume $B$ is an $\omega$-d $\mathbf{d}^{p}$-saturated model. By Theorem 6.4 or 6.3 there exists a separable $A \subset B$ such that $a \downarrow_{A}^{s} B$ and $b \downarrow_{A}^{s} B$, and again by Lemma 5.2 and monotonicity we may assume $A$ is $\omega$-d $\mathbf{d}^{p}$-saturated and closed in $B$. Then by stationarity (Theorem 6.6) $\mathrm{t}^{g}(a / B)=\mathrm{t}^{g}(b / B)$ if and only if $\mathrm{t}^{g}(a / A)=$ $\mathrm{t}^{g}(b / A)$. Hence to count the types over $B$ it suffices to count the number of types over separable closed subsets of $B$. Since $\lambda^{\aleph_{0}}=\lambda$, there are only $\lambda$ such subsets of $B$. Further types over separable sets are determined by their restrictions to some dense (countable) subset and there are only $2^{\aleph_{0}}$ types over countable sets (recall that the vocabulary is countable) so there are just $\lambda$ types over $B$.

Since our stability notion in a sense considers weak types, our perturbation assumption gives a property that resembles the metric homogeneity assumption from [9]. As might be expected we get similar stability results 
as in [9] and below we prove that $\omega$ - $\mathbf{d}^{p}$-stability implies $\lambda$-d $\mathbf{d}^{p}$-stability for all infinite $\lambda$. It is, however, worth noting, that we do not assume metric homogeneity, which considers the orbits of Galois types over fixed parameter sets.

Theorem 6.9. If $\mathbb{K}$ is $\omega$-d $\mathbf{d}^{p}$-stable and has complete type-spaces or good models (i.e. $(\mathcal{A}, \mathcal{A})$ is good for all $\mathcal{A} \in \mathbb{K})$ then it is $\lambda$-d $\mathbf{d}^{p}$-stable for all infinite $\lambda$.

Proof. Assume towards a contradiction that $\mathbb{K}$ is not $\lambda$-stable. Then there is $B \subset \mathfrak{M}$ with $|B|=\lambda$ such that the density of $S(B)$ with respect to $\mathbf{d}^{p}$ is at least $\lambda^{+}$and by Lemma 5.2 we may assume $B$ is an $\omega$-d $\mathbf{d}^{p_{\text {-saturated }}}$ model. Now there are $a_{i}, i<\lambda^{+}$and some $\varepsilon>0$ such that

$$
\mathbf{d}^{p}\left(\mathrm{t}^{g}\left(a_{i} / B\right), \mathrm{t}^{g}\left(a_{j} / B\right)\right) \geq \varepsilon
$$

for all $i<j<\lambda^{+}$.

Let $\varepsilon^{\prime}=\Delta^{\varepsilon}(\varepsilon / 6)$. For each $i<\lambda^{+}$, by either Theorem 6.3 or 6.4 , choose a finite $A_{i} \subset B$ and a rational $\delta_{i}>0$ such that $\mathrm{t}^{g}\left(a_{i} / B\right)$ does not $\varepsilon^{\prime}$-split over $A_{i}$ and $\delta_{i}$ witnesses this. $\lambda^{+}$many of the pairs $\left(A_{i}, \delta_{i}\right)$ are the same, denote these by $A$ and $\delta$. Next, by Lemma 5.2 let $\mathcal{A} \preccurlyeq B$ be separable, $\omega$-d ${ }^{p}$-saturated and contain $A$.

Now for $i \neq j$, since

$$
\mathbf{d}^{p}\left(\mathrm{t}^{g}\left(a_{i} / B\right), \mathrm{t}^{g}\left(a_{j} / B\right)\right)=\sup \left\{\mathbf{d}^{p}\left(\mathrm{t}^{g}\left(a_{i} c / \emptyset\right), \mathrm{t}^{g}\left(a_{j} c / \emptyset\right)\right): c \in B \text { finite }\right\},
$$

there is some finite $c_{i j} \in B$ such that

$$
\mathbf{d}^{p}\left(\mathrm{t}^{g}\left(a_{i} c_{i j} / \emptyset\right), \mathrm{t}^{g}\left(a_{j} c_{i j} / \emptyset\right)\right)>\varepsilon / 2 .
$$

For all $i \neq j$ such that $\left(A_{i}, \delta_{i}\right)=\left(A_{j}, \delta_{j}\right)=(A, \delta)$, let $c^{\prime} \in \mathcal{A}$ be such that $\mathbf{d}^{p}\left(\mathrm{t}^{g}\left(c_{i j} / A\right), \mathrm{t}^{g}\left(c^{\prime} / A\right)\right) \leq \delta$. Then we must have

$$
\mathbf{d}^{p}\left(\mathrm{t}^{g}\left(a_{i} / c^{\prime} A\right), \mathrm{t}^{g}\left(a_{j} / c^{\prime} A\right)\right)>\varepsilon^{\prime}
$$

since by non-splitting $\mathbf{d}^{p}\left(\mathrm{t}^{g}\left(a_{i} c_{i j} / A\right), \mathrm{t}^{g}\left(a_{i} c^{\prime} / A\right)\right) \leq \varepsilon^{\prime}$ (and similarly for $a_{j}$ ) and combining this with (6.5) would give

$$
\mathbf{d}^{p}\left(\mathrm{t}^{g}\left(a_{i} c_{i j} / A\right), \mathrm{t}^{g}\left(a_{j} c_{i j} / A\right)\right) \leq 3 \varepsilon / 6,
$$

a contradiction. But then we have $\mathbf{d}^{p}\left(\mathrm{t}^{g}\left(a_{i} / \mathcal{A}\right), \mathrm{t}^{g}\left(a_{j} / \mathcal{A}\right)\right)>\varepsilon^{\prime}$ for $\lambda^{+}$many $i \neq j$, contradicting $\omega$ - $\mathbf{d}^{p}$-stability.

Next we will tie our notion of independence to the usual one in homogeneous model theory. In [9] it is shown how to put the abstract setting of homogeneous metric abstract elementary classes into a homogeneous first 
order context hence gaining access to results from [10] (as long as we work in a stable class).

We recall some definitions from [10], as stated in a formula-free fashion in [9].

Definition 6.10. (1) Let $A \subset B$ and $a \in \mathfrak{M}$. We say that $\mathrm{t}^{g}(a / B)$ splits over $A$ if there are $b, c \in B$ such that $\mathrm{t}^{g}(b / A)=\mathrm{t}^{g}(c / A)$ but $\mathrm{t}^{g}(b / A \cup a) \neq \mathrm{t}^{g}(c / A \cup a)$.

(2) A type $\mathrm{t}^{g}(a / B)$ is said to split strongly over $A \subset B$ if there are $b, c \in B$ and an infinite sequence $I$, indiscernible over $A$, with $b, c \in I$ such that $\mathrm{t}^{g}(b / A \cup a) \neq \mathrm{t}^{g}(c / A \cup a)$.

(3) $\kappa(\mathbb{K})$ denotes the least cardinal such that there are no $a, b_{i}$ and $c_{i}$ for $i<\kappa(\mathbb{K})$ such that $\mathrm{t}^{g}\left(a / \bigcup_{j \leq i}\left(b_{j} \cup c_{j}\right)\right)$ splits strongly over $\bigcup_{j<i}\left(b_{j} \cup c_{j}\right)$ for each $i<\kappa(\mathbb{K})$.

(4) $a \downarrow_{A} B$ if there is $C \subseteq A$ of cardinality $<\kappa(\mathfrak{M})$ such that for all $D \supseteq A \cup B$ there is $b$ with $\mathrm{t}^{g}(b / A \cup B)=\mathrm{t}^{g}(a / A \cup B)$ such that $\mathrm{t}^{g}(b / D)$ does not split strongly over $C$.

(5) $\lambda(\mathfrak{M})$ is the least cardinality in which $\mathfrak{M}$ is stable.

Remark 6.11. Note that if $a \downarrow_{A}^{s} B$ then by perturbation $\mathrm{t}^{g}(a / A \cup B)$ does not split over $A$ and hence it does not split strongly over $A$.

Now the equivalence of $\downarrow$ and $\downarrow^{s}$ over saturated enough models can be proved just as in [9].

Theorem 6.12. Assume $\mathbb{K}$ is $\omega$-d $\mathbf{d}^{p}$-stable and has complete type-spaces. Then if $\mathcal{A} \subseteq B, a \in \mathfrak{M}$ and $\mathcal{A}$ is $2^{\aleph_{0}}$-saturated (i.e. realizes all Galois types over parameter sets of size $<2^{\aleph_{0}}$ ) then

$$
a \downarrow_{\mathcal{A}}^{s} B \text { if and only if } a \downarrow_{\mathcal{A}} B,
$$

i.e. our notion of independence agrees with that in classical homogeneous model theory over $2^{\aleph_{0}}$-saturated models.

Proof. First assume $a \downarrow_{\mathcal{A}} B$. By local character (Theorem 6.6) $a \downarrow_{\mathcal{A}}^{s} \mathcal{A}$. Let $\mathcal{B} \supseteq B$ be $2^{\aleph_{0}}$-saturated and by extension (Theorem 6.6) find some $b$ realizing $\mathrm{t}^{g}(a / \mathcal{A})$ and satisfying $b \downarrow_{\mathcal{A}}^{s} \mathcal{B}$. Then $\mathrm{t}^{g}(b / \mathcal{B})$ does not split strongly over $\mathcal{A}$ so by [10, Lemma 3.2.(iii)] $b \downarrow_{\mathcal{A}} \mathcal{B}$ and by monotonicity $b \downarrow_{\mathcal{A}} B$. Then by stationarity of $\downarrow$ ([10, Lemma 3.4]), $\mathrm{t}^{g}(b / B)=\mathrm{t}^{g}(a / B)$ so $a \downarrow_{A}^{s} B$.

For the other direction assume $a \downarrow_{\mathcal{A}}^{s} B$. Again by [10, Lemma 3.2(iii)], $a \downarrow_{\mathcal{A}} \mathcal{A}$. Since $\downarrow$ has built in extensions this implies the existence of some 
$b$ realizing $\mathrm{t}^{g}(a / \mathcal{A})$ and satisfying $b \downarrow_{\mathcal{A}} B$. Then by the previous direction $b \downarrow_{\mathcal{A}}^{s} B$ so by stationarity of $\downarrow^{s}$ we are done.

Then using symmetry of $\downarrow$ over so-called a-saturated (strongly $\kappa(\mathfrak{M})$ saturated) models ([10, Lemma 3.6]) we can prove symmetry for $\downarrow^{s}$. We use the fact ([10, Lemma 1.9(iv)]) that $\lambda(\mathfrak{M})$-saturated models are a-saturated and recall that by Lemma $6.8 \lambda(\mathfrak{M})$ is at most $2^{\aleph_{0}}$, when $\mathbb{K}$ is $\omega$-d $\mathbf{d}^{p}$-stable and has complete type-spaces.

Lemma 6.13. If $\mathbb{K}$ is $\omega$-d $\mathbf{d}^{p}$-stable and has complete type-spaces, $A$ is $\omega$-d $\mathbf{d}^{p}$ saturated and $a \downarrow_{A}^{s} b$ then $b \downarrow_{A}^{s} a$.

Proof. Assume towards a contradiction that $a \downarrow_{A}^{s} b$ but $b \quad \chi_{A}^{s} a$. Let $\mathcal{B} \supset A$ be $2^{\aleph_{0}}$-saturated. Now $b \downarrow_{A}^{s} A$ so by the extension property (Theorem 6.6) there is some $b^{\prime}$ realizing $\mathrm{t}^{g}(b / A)$ and satisfying $b^{\prime} \downarrow_{A}^{s} \mathcal{B}$. We may assume $b^{\prime}=b$ (since moving $\mathcal{B}$ by a 0 -automorphism does not affect its degree of saturation). Using the extension property and stationarity, we can find some $a^{\prime}$ realizing $\mathrm{t}^{g}(a / A b)$ and satisfying $a^{\prime} \downarrow_{A}^{s} \mathcal{B} b$. Now by monotonicity we have $a^{\prime} \downarrow_{\mathcal{B}}^{s} b$. But we cannot have $b \downarrow_{\mathcal{B}}^{s} a^{\prime}$ since then by transitivity we would have $b \downarrow_{A}^{s} \mathcal{B} a^{\prime}$, by monotonicity $b \downarrow_{A}^{s} a^{\prime}$ and hence by invariance $b \downarrow_{A}^{s} a$. Hence $a^{\prime} \downarrow_{\mathcal{B}}^{s} b$ and $b \quad \chi_{\mathcal{B}}^{s} a^{\prime}$, but since $\mathcal{B}$ is $2^{\aleph_{0}}$-saturated, by Lemma 6.12 this contradicts symmetry of $\downarrow$ ([10, Lemma 3.6]) over a-saturated models.

\section{ISOLATION}

In this section we develop a notion of isolation and build constructible sets. With our notion of isolation dominance works, but whether our constructible sets are prime in the class of $\omega$-d $\mathbf{d}^{p}$-saturated models in any reasonable sense is an open question. We will discuss this matter at the end of the section.

Definition 7.1. We say that $\mathrm{t}^{g}(a / A)$ is $\varepsilon$-isolated if there are some $\delta>0$ and finite $A^{\prime} \subset A$ such that $\mathbf{d}^{p}\left(\mathrm{t}^{g}\left(b / A^{\prime}\right), \mathrm{t}^{g}\left(a / A^{\prime}\right)\right) \leq \delta$ implies

$$
\mathbf{d}^{p}\left(\mathrm{t}^{g}(b / A), \mathrm{t}^{g}(a / A)\right) \leq \varepsilon .
$$

We say that $\mathrm{t}^{g}(a / A)$ is isolated if it is $\varepsilon$-isolated for all $\varepsilon>0$.

The next lemma follows also from [2, Proposition 3.7].

Lemma 7.2. If $\mathbb{K}$ is $\omega$-d $\mathbf{d}^{p}$-stable and has complete type-spaces then for all $A, a, \varepsilon>0, \delta>0$ and finite $B \subset A$ there is some $a^{\prime}$ with

$$
\mathbf{d}^{p}\left(\mathrm{t}^{g}\left(a^{\prime} / B\right), \mathrm{t}^{g}(a / B)\right) \leq \delta
$$


such that $\mathrm{t}^{g}\left(a^{\prime} / A\right)$ is $\varepsilon$-isolated.

Proof. Assume towards a contradiction that the claim is false. Then for every $a^{\prime}$ with $\mathbf{d}^{p}\left(\mathrm{t}^{g}\left(a^{\prime} / B\right), \mathrm{t}^{g}(a / B)\right) \leq \delta$ and for all $\delta^{\prime}>0$ and finite $A^{\prime} \subset A$ there is some $b$ such that $\mathbf{d}^{p}\left(\mathrm{t}^{g}\left(b / A^{\prime}\right), \mathrm{t}^{g}\left(a^{\prime} / A^{\prime}\right)\right) \leq \delta^{\prime}$ but

$$
\mathbf{d}^{p}\left(\mathrm{t}^{g}(b / A), \mathrm{t}^{g}\left(a^{\prime} / A\right)\right)>\varepsilon .
$$

Let $0<\delta^{\prime} \leq \min \left\{\delta / 4, \Delta^{\delta}(\varepsilon / 5)\right\}$. Then define, for $n<\omega$ and $\xi \in 2^{<\omega}$, $A_{n} \subset A, \delta_{n}>0, A_{\xi} \subset A$ and $a_{\xi}$ such that

- $A_{0}=B, A_{n} \subseteq A_{n+1}, A_{n}$ is finite,

- $\delta_{n+1} \leq \delta_{n}$ and $\sum_{n<\omega} \delta_{n} \leq \delta^{\prime}$,

- $a_{\emptyset}=a$ and $a_{\xi \wedge(0)}=a_{\xi}$,

- $\mathrm{d}^{p}\left(\mathrm{t}^{g}\left(a_{\xi} / B\right), \mathrm{t}^{g}(a / B)\right) \leq \sum_{i<\operatorname{length}(\xi)} \delta_{i}<\delta^{\prime}$

- $\mathrm{d}^{p}\left(\mathrm{t}^{g}\left(a_{\xi \frown(0)} / A_{\text {length }(\xi)}\right), \mathrm{t}^{g}\left(a_{\xi \frown(1)} / A_{\text {length }(\xi)}\right)\right) \leq \Delta^{\delta^{\prime}}\left(\delta_{\text {length }(\xi)}\right)$ but

$$
\mathbf{d}^{p}\left(\mathrm{t}^{g}\left(a_{\xi \frown(0)} / A_{\xi}\right), \mathrm{t}^{g}\left(a_{\xi \frown(1)} / A_{\xi}\right)\right)>\varepsilon,
$$

- $A_{n+1}=\bigcup_{\text {length }(\xi)=n} A_{\xi}$.

When length $(\xi)=n$ and $A_{n}$ and $a_{\xi}$ have been defined such that

$$
\mathbf{d}^{p}\left(\mathrm{t}^{g}\left(a_{\xi} / B\right), \mathrm{t}^{g}(a / B)\right) \leq \sum_{i<n} \delta_{i}
$$

then by our counter-assumption we may find some $a_{\xi \sim(1)}$ satisfying

$$
\mathbf{d}^{p}\left(\mathrm{t}^{g}\left(a_{\xi} / A_{n}\right), \mathrm{t}^{g}\left(a_{\xi \frown(1)} / A_{n}\right)\right) \leq \Delta^{\delta^{\prime}}\left(\delta_{n}\right)
$$

and

$$
\mathbf{d}^{p}\left(\mathrm{t}^{g}\left(a_{\xi} / A\right), \mathrm{t}^{g}\left(a_{\xi \sim(1)} / A\right)\right)>\varepsilon .
$$

Then we let $A_{\xi} \subset A$ be finite and witness (7.2) and note that (7.1) implies

$$
\mathbf{d}^{p}\left(\mathrm{t}^{g}\left(a_{\xi \frown(1)} / B\right), \mathrm{t}^{g}(a / B)\right) \leq \sum_{i<n+1} \delta_{i} \leq \delta^{\prime}
$$

keeping the induction going.

Next note that for each $\eta \in{ }^{\omega} 2,\left\{\mathrm{t}^{g}\left(a_{\eta\lceil n} / A_{n}\right): n<\omega\right\}$ forms a $\mathbf{d}^{p}$-coherent system in the sense of Lemma 4.6. Hence there is for each $\eta \in{ }^{\omega} 2$ a limit element $a_{\eta}$. But now for $\eta \neq \nu, \eta, \nu \in{ }^{\omega} 2$,

$$
\mathbf{d}^{p}\left(\mathrm{t}^{g}\left(a_{\eta} / \bigcup_{n<\omega} A_{n}\right), \mathrm{t}^{g}\left(a_{\nu} / \bigcup_{n<\omega} A_{n}\right)\right)>\delta^{\prime}
$$

since otherwise, when $n=\min \{n: \eta(n) \neq \nu(n)\}$ and $N$ large enough,

- $\mathbf{d}^{p}\left(\mathrm{t}^{g}\left(a_{\eta\lceil n+1} / A_{n+1}\right), \mathrm{t}^{g}\left(a_{\eta \uparrow N} / A_{n+1}\right)\right) \leq \delta^{\prime}$ (and similarly for $\nu$ ) by construction, 
- $\mathrm{d}^{p}\left(\mathrm{t}^{g}\left(a_{\eta \uparrow N} / A_{n+1}\right), \mathrm{t}^{g}\left(a_{\eta} / A_{n+1}\right)\right) \leq \delta^{\prime}$ (and similarly for $\nu$ ) by choice of $N$ and

- $\mathbf{d}^{p}\left(\mathrm{t}^{g}\left(a_{\eta} / A_{n+1}\right), \mathrm{t}^{g}\left(a_{\nu} / A_{n+1}\right)\right) \leq \delta^{\prime}$

and combining gives

$$
\mathbf{d}^{p}\left(\mathrm{t}^{g}\left(a_{\eta\lceil n+1} / A_{n+1}\right), \mathrm{t}^{g}\left(a_{\nu\lceil n+1} / A_{n+1}\right)\right) \leq \varepsilon,
$$

contradicting the way we chose the $a_{\xi}$ s.

But (7.3) contradicts $\omega$-d $\mathbf{d}^{p}$-stability since $\bigcup_{n<\omega} A_{n}$ is countable.

Theorem 7.3. If $\mathbb{K}$ is $\omega$-d $\mathbf{d}^{p}$-stable and has complete type-spaces then for all $A, a, \delta>0$ and finite $B \subset A$ there is some $a^{\prime}$ with $\mathbf{d}^{p}\left(\mathrm{t}^{g}\left(a^{\prime} / B\right), \mathrm{t}^{g}(a / B)\right) \leq \delta$ such that $\mathrm{t}^{g}(b / A)$ is isolated.

Proof. By the previous lemma we can, for each positive $n<\omega$ and $\delta_{n}^{\prime}$ find finite sets $A_{n} \subset A, \delta_{n}>0$ and elements $a_{n}$ such that $A_{n}$ and $\delta_{n} \frac{1}{n}$-isolate $\mathrm{t}^{g}\left(a_{n} / A\right)$ and $\mathbf{d}^{p}\left(\mathrm{t}^{g}\left(a_{n+1} / A_{n}\right), \mathrm{t}^{g}\left(a_{n} / A_{n}\right)\right) \leq \delta_{n+1}^{\prime}$. We just need to make sure that all $a_{i}$ for $i>n$ are within distance $\delta_{n}$ of $a_{n}$.

So we define

- $A_{0}=B, a_{0}=a, \delta_{0}^{\prime}=\delta_{0}=\delta / 2$,

- $A_{n} \subset A_{n+1} \subset A, A_{n}$ is finite and for $n>0, \delta_{n}$ and $A_{n} \frac{1}{n}$-isolate $\mathrm{t}^{g}\left(a_{n} / A\right)$,

- $\delta_{n+1}^{\prime} \leq \min \left\{\Delta^{\delta}\left(\delta_{n} / 4\right), \delta_{n}^{\prime} / 4\right\}$,

- $\mathbf{d}^{p}\left(\mathrm{t}^{g}\left(a_{n+1} / A_{n}\right), \mathrm{t}^{g}\left(a_{n} / A_{n}\right)\right) \leq \Delta^{\delta}\left(\delta_{n+1}^{\prime}\right)$.

Then $\left\{\mathrm{t}^{g}\left(a_{n} / A_{n}: n<\omega\right\}\right.$ forms a $\mathbf{d}^{p}$-coherent system in the sense of Lemma 4.6 and hence there is a limit element $a^{\prime}$ such that

$$
\mathbf{d}^{p}\left(\mathrm{t}^{g}\left(a^{\prime} / A_{n}\right), \mathrm{t}^{g}\left(a_{n} / A_{n}\right)\right) \leq \Delta^{\delta}\left(\delta_{n} / 2\right)
$$

for each $n<\omega$. Hence especially $\mathbf{d}^{p}\left(\mathrm{t}^{g}\left(a^{\prime} / B\right), \mathrm{t}^{g}(a / B)\right) \leq \delta$ and it is fairly straightforward to see that if $\frac{1}{n} \leq \Delta^{\varepsilon}(\varepsilon)$ for a given $\varepsilon>0$ then $A_{n}$ and $\Delta^{\delta}\left(\delta_{n} / 2\right)$ Eisolate $\mathrm{t}^{g}\left(a^{\prime} / A\right)$.

Definition 7.4. We say that $A^{*}$ is constructible over $A$ if $A^{*}=A \cup\left\{a_{i}\right.$ : $i<\alpha\}$ and for each $i<\alpha, \mathrm{t}^{g}\left(a_{i} / A \cup\left\{a_{j}: j<i\right\}\right.$ is isolated.

Remark 7.5. Note that if $B$ is constructible over $A$ then $\bar{B}$ is also constructible over $A$, since any element from $B$ close enough to $b \in \bar{B}$ isolates the type of $b$ over any set.

Theorem 7.6. If $\mathbb{K}$ is $\omega$-d $\mathbf{d}^{p}$-stable and has complete type-spaces then for each $A$ there is a complete $\omega$-d $\mathbf{d}^{p}$-saturated constructible set over $A$. 
Proof. This is done more or less in the standard way. If we have constructed $A_{\alpha}=A \cup\left\{a_{i}: i<\alpha\right\}$ and $A_{\alpha}$ is not $\omega$-d $\mathbf{d}^{p}$-saturated then there is some finite $A^{\prime} \subset A_{\alpha}$, some $a$ and $\delta>0$ such that no $a^{\prime} \in A_{\alpha}$ satisfies $\mathbf{d}^{p}\left(\mathrm{t}^{g}\left(a^{\prime} / A^{\prime}\right), \mathrm{t}^{g}\left(a / A^{\prime}\right)\right) \leq \delta$. But by Theorem 7.3 we can find some $a^{\prime}$ such that $\mathrm{t}^{g}\left(a^{\prime} / A_{\alpha}\right)$ is isolated and $\mathbf{d}^{p}\left(\mathrm{t}^{g}\left(a^{\prime} / A^{\prime}\right), \mathrm{t}^{g}\left(a / A^{\prime}\right)\right) \leq \delta$ and then let $a_{\alpha}=a^{\prime}$. The construction will eventually terminate at some $\alpha$ (essentially as in [12, Theorem IV.3.1]) giving a constructible $\omega$-d $\mathbf{d}^{p}$-saturated set $A_{\alpha}$. However, $A_{\alpha}$ need not be complete, but by Remark 7.5 we may continue the construction until some $\alpha^{\prime} \geq \alpha$ such that $A_{\alpha^{\prime}}=\overline{A_{\alpha}}$, and by Remark 5.3 $A_{\alpha^{\prime}}$ is still $\omega$-d ${ }^{p}$-saturated.

Definition 7.7. $B \supset A$ is atomic over $A$ if for each $b \in B, \mathrm{t}^{g}(b / A)$ is isolated.

Theorem 7.8. If $B$ is constructible over $A$ then $B$ is atomic over $A$.

Proof. This is done by a fairly straightforward induction on $i<\alpha$, where $B=A \cup\left\{a_{i}: i<\alpha\right\}$ and each $\mathrm{t}^{g}\left(a_{i} / A \cup\left\{a_{j}: j<i\right\}\right)$ is isolated.

Theorem 7.9. Assume $\mathbb{K}$ is $\omega$-d $\mathbf{d}^{p}$-stable and has complete type-spaces. If $A$ is $\omega$-d $\mathbf{d}^{p}$-saturated, $B \supseteq A$ and $B^{*}$ is constructible over $B$ then $a \downarrow_{A}^{s} B$ implies $a \downarrow_{A}^{s} B^{*}$.

Proof. Assume towards a contradiction that $a \downarrow_{A}^{s} B$ but $a \quad \chi_{A}^{s} B^{*}$. By countable character (Theorem 6.6) there is a finite $b \in B^{*}$ such that $a \quad \chi_{A}^{s} b$. Further by symmetry $b \quad \chi_{A}^{s} a$, so there is some $\varepsilon>0$ such that $b \quad \chi_{A}^{\varepsilon} a$, i.e. $\mathrm{t}^{g}(b / A a) \varepsilon$-splits over every finite $A^{\prime} \subset A$.

Let $\varepsilon^{\prime}<\Delta^{\varepsilon}(\varepsilon / 2)$ and $\varepsilon^{\prime \prime}<\Delta^{\varepsilon^{\prime}}\left(\varepsilon^{\prime} / 2\right)$.

By Theorem $7.8 B^{*}$ is atomic over $B$ so we can find some finite $B^{\prime} \subset B$ and $\delta^{\prime}>0$, with $\delta^{\prime} \leq \varepsilon^{\prime \prime}$ such that $B^{\prime}$ and $\delta^{\prime}$ witness the $\varepsilon^{\prime \prime}$-isolation of $\mathrm{t}^{g}(b / B)$. Then let $\delta^{\prime \prime}<\Delta^{\varepsilon}\left(\delta^{\prime} / 2\right)$. Since $a \downarrow_{A}^{s} B$, we have $a \downarrow_{A}^{s} B^{\prime}$ and again by symmetry $B^{\prime} \downarrow_{A}^{s} a$. Hence there is some finite $A^{\prime} \subset A$ such that $\mathrm{t}^{g}\left(B^{\prime} / A a\right)$ does not $\delta^{\prime \prime}$-split over $A^{\prime}$ and there is some $\delta^{\prime \prime \prime}>0$ witnessing this. Now since $b \quad \chi_{A}^{\varepsilon} a$ there are $c, c^{\prime} \in A \cup a$ such that $\mathbf{d}^{p}\left(\mathrm{t}^{g}\left(c / A^{\prime}\right), \mathrm{t}^{g}\left(c^{\prime} / A^{\prime}\right)\right) \leq \delta^{\prime \prime \prime}$ but

$$
\mathbf{d}^{p}\left(\mathrm{t}^{g}\left(b c / A^{\prime}\right), \mathrm{t}^{g}\left(b c^{\prime} / A^{\prime}\right)\right)>\varepsilon .
$$

Since $\delta^{\prime \prime \prime}$ witnesses the fact that $\mathrm{t}^{g}\left(B^{\prime} / A a\right)$ does not $\delta^{\prime \prime}$-split over $A^{\prime}$, we must have $\mathbf{d}^{p}\left(\mathrm{t}^{g}\left(B^{\prime} c / A^{\prime}\right), \mathrm{t}^{g}\left(B^{\prime} c^{\prime} / A^{\prime}\right)\right) \leq \delta^{\prime \prime}$. So there is $f \in$ Aut $_{\delta^{\prime \prime}}$ such that

$$
\mathrm{d}\left(f\left(B^{\prime} c A^{\prime}\right), B^{\prime} c^{\prime} A^{\prime}\right) \leq \delta^{\prime \prime} .
$$


Case 1: $c^{\prime} \in A$ (or $c \in A$, but that case is treated symmetrically). Now $\mathbf{d}^{p}\left(\mathrm{t}^{g}\left(b / B^{\prime}\right), \mathrm{t}^{g}\left(f(b) / B^{\prime}\right)\right) \leq \delta^{\prime \prime}$ so by the $\varepsilon^{\prime \prime}$-isolation of $\mathrm{t}^{g}(b / B)$

$$
\mathbf{d}^{p}\left(\mathrm{t}^{g}(b / B), \mathrm{t}^{g}(f(b) / B)\right) \leq \varepsilon^{\prime \prime} .
$$

So (since $A \subseteq B$ ) there is $g \in$ Aut $_{\varepsilon^{\prime \prime}}$ satisfying $\mathrm{d}\left(g\left(f(b) c^{\prime} A^{\prime}\right), b c^{\prime} A^{\prime}\right) \leq \varepsilon^{\prime \prime}$ and as

$$
\mathrm{d}\left(g \circ f\left(b c A^{\prime}\right), b c^{\prime} A^{\prime}\right) \leq \mathrm{d}\left(g \circ f\left(b c A^{\prime}\right), g\left(f(b) c^{\prime} A^{\prime}\right)\right)+\mathrm{d}\left(g\left(f(b) c^{\prime} A^{\prime}\right), b c^{\prime} A^{\prime}\right)
$$

we have

$$
\mathbf{d}^{p}\left(\mathrm{t}^{g}\left(b c / A^{\prime}\right), \mathrm{t}^{g}\left(b c^{\prime} / A^{\prime}\right)\right) \leq 2 \varepsilon^{\prime \prime}<\varepsilon,
$$

contradicting the choice of $c$ and $c^{\prime}$.

Case 2: $c, c^{\prime} \notin A$. Since $A$ is $\omega$-d $\mathbf{d}^{p}$-saturated, there is $d^{\prime} \in A$ such that $\mathbf{d}^{p}\left(\mathrm{t}^{g}\left(c^{\prime} / A^{\prime}\right), \mathrm{t}^{g}\left(d^{\prime} / A^{\prime}\right)\right) \leq \delta^{\prime \prime \prime}$, and again by non- $\delta^{\prime \prime}$-splitting of $\mathrm{t}^{g}\left(B^{\prime} / A a\right)$ we may choose some $g \in \operatorname{Aut}_{\delta^{\prime \prime}}$ with $\mathrm{d}\left(g\left(B^{\prime} c^{\prime} A^{\prime}\right), B^{\prime} d^{\prime} A^{\prime}\right) \leq \delta^{\prime \prime}$. Then $\mathbf{d}^{p}\left(\mathrm{t}^{g}(g \circ\right.$ $\left.\left.f(b) / B^{\prime}\right), \mathrm{t}^{g}\left(b / B^{\prime}\right)\right) \leq \delta^{\prime}$. So by $\varepsilon^{\prime \prime}$-isolation, $\mathbf{d}^{p}\left(\mathrm{t}^{g}(g \circ f(b) / B), \mathrm{t}^{g}(b / B)\right) \leq$ $\varepsilon^{\prime \prime}$. Also $\mathbf{d}^{p}\left(\mathrm{t}^{g}(b / B), \mathrm{t}^{g}(g(b) / B)\right) \leq \varepsilon^{\prime \prime}$ (since $\left.\mathbf{d}^{p}\left(\mathrm{t}^{g}\left(b / B^{\prime}\right), \mathrm{t}^{g}\left(g(b) / B^{\prime}\right)\right) \leq \delta^{\prime}\right)$. Hence $\mathbf{d}^{p}\left(\mathrm{t}^{g}(g \circ f(b) / B), \mathrm{t}^{g}(g(b) / B)\right) \leq \varepsilon^{\prime}$ so letting $h \in$ Aut $_{\varepsilon^{\prime}}$ witness this we have $g^{-1} \circ h \circ g \circ f \in$ Aut $\left._{2 \varepsilon^{\prime}}\right)$ and

$$
\begin{aligned}
& \mathrm{d}\left(g^{-1} \circ h \circ g \circ f\left(b c A^{\prime}\right), b c^{\prime} A^{\prime}\right) \\
& \leq \quad \mathrm{d}\left(g^{-1} \circ h \circ g \circ f\left(b c A^{\prime}\right), g^{-1} \circ h \circ g\left(f(b) c^{\prime} A^{\prime}\right)\right) \\
& \quad+\mathrm{d}\left(g^{-1} \circ h \circ g\left(f(b) c^{\prime} A^{\prime}\right), g^{-1} \circ h\left(g \circ f(b) d^{\prime} A^{\prime}\right)\right) \\
& \quad+\mathrm{d}\left(g^{-1} \circ h\left(g \circ f(b) d^{\prime} A^{\prime}\right), g^{-1}\left(g(b) d^{\prime} A^{\prime}\right)\right) \\
& \quad+\mathrm{d}\left(g^{-1}\left(g(b) d^{\prime} A^{\prime}\right), b c^{\prime} A^{\prime}\right) \\
& \leq \varepsilon
\end{aligned}
$$

and thus $\mathbf{d}^{p}\left(\mathrm{t}^{g}\left(b c / A^{\prime}\right), \mathrm{t}^{g}\left(b c^{\prime} / A^{\prime}\right)\right) \leq \varepsilon$ again contradicting the choice of $c$ and $c^{\prime}$.

We now return to the question about the difficulties in proving the existence of prime models for the class of $\omega$-d $\mathbf{d}^{p}$-saturated models.

In classical model theory results like this are proved in the following way: Suppose $*$-saturation is some notion of saturation and $F$ is some reasonable isolation notion. To find prime models for the class of $*$-saturated models one needs to prove the following two properties:

(1) Every $F$-isolated type over $A$ can be extended to an $F$-isolated type over $B \supset A$.

(2) $\mathcal{A}$ is $*$-saturated if and only if $\mathcal{A}$ is $F$-saturated, i.e. every $F$-isolated type over $\mathcal{A}$ is realized in $\mathcal{A}$. 
In our context we can prove $\mathbf{d}^{p}$-versions of 1 and 2 (see remark below) but we do not seem to be able to show that the properties imply the existence of prime models in any reasonable sense for the class of $\omega$-d $\mathbf{d}^{p}$-saturated models. Note that if $\mathbf{d}^{p}$ is replaced by $\mathbf{d}$, the standard metric on types defined as the infimum of distances of realizations, this still works, as seen in [9].

Remark 7.10. Assume $\mathbb{K}$ is $\omega$ - $\mathbf{d}^{p}$-stable and has complete type-spaces.

(1) If $\mathrm{t}^{g}(a / A)$ is isolated and $B \supseteq A$ then for all $\varepsilon>0$ there is $b$ such that $\mathrm{t}^{g}(b / B)$ is isolated and $\mathbf{d}^{p}\left(\mathrm{t}^{g}(b / A), \mathrm{t}^{g}(a / A)\right) \leq \varepsilon$.

(2) If $A$ is $\omega$-d $\mathbf{d}^{p}$-saturated and complete and $\mathrm{t}^{g}(a / A)$ is isolated then $a \in A$.

(3) If $A$ realizes every isolated type over $A$ then $A$ is $\omega$-d $\mathbf{d}^{p}$-saturated and complete.

Proof. 1 and 3 are immediate consequences of Theorem 7.3. For 2 prove that the distance of $a$ to the set $A$ is less than any given $\varepsilon$, by using $\omega$-d $\mathbf{d}^{p}$ saturation over a suitably isolating set.

The problem here lies in the first item which forces us to "switch type" as we want to extend the isolation. Note that without this type-switching the item does not hold: otherwise $\omega$-d $\mathbf{d}^{p}$-saturated models would be $\omega$-saturated. But considering the class of $L^{p}$-spaces with $\mathbb{F}_{\varepsilon}=\mathbb{F}_{0}$ for all $\varepsilon$, this would contradict Ben Yaacov's and Usvyatsov's observation in [5] that $L^{p}[0,1]$ is not $\omega$-saturated.

We finish this paper by giving an example on how to use the theory developed in this paper. Lemma 7.11 below is a general result that implies that the class $\mathbb{K}_{H}$ from section 3 has the maximal number of models in density character $\omega_{1}$ (choose $\mathcal{A}$ to be any existentially closed model and $a_{i}$ for $i<\omega_{1}$ to be any non-zero element such that $a_{i}$ is orthogonal to $\mathcal{A}$ in the sense of Hilbert spaces and $\tau a_{i}=q_{i} a_{i}$, where $q_{i}, i<\omega_{1}$, are distinct complex numbers with norm 1 ). The proof is similar to the first order proof that non-unidimensional theories are not categorical. Notice that the class $\mathbb{K}$ in Lemma 7.11 may be unsuperstable in the ordinary metric sense and stability alone does not allow one to make constructions like the one in the lemma.

We say that $\mathrm{t}^{g}(a / \emptyset)$ is orthogonal to $\mathrm{t}^{g}(b / \emptyset)$ if for all $\omega$-d $\mathbf{d}^{p}$-saturated $\mathcal{A}$, $a^{\prime}$ and $b^{\prime}$ if $\mathrm{t}^{g}\left(a^{\prime} / \emptyset\right)=\mathrm{t}^{g}(a / \emptyset), \mathrm{t}^{g}\left(b^{\prime} / \emptyset\right)=\mathrm{t}^{g}(b / \emptyset), a^{\prime} \downarrow_{\emptyset}^{s} \mathcal{A}$ and $b^{\prime} \downarrow_{\emptyset}^{s} \mathcal{A}$, then $a^{\prime} \downarrow_{\mathcal{A}}^{s} b^{\prime}$. 
Lemma 7.11. Suppose $\mathbb{K}$ is $\omega$-d $\mathbf{d}^{p}$-stable, has a complete type space and every complete $\omega$-d $\mathbf{d}^{p}$-saturated set is a model. Assume that there are $\omega-\mathbf{d}^{p}$ saturated $\mathcal{A}$ and $a_{i} \notin \mathcal{A}, i<\omega_{1}$, such that $a_{i} \downarrow_{\emptyset}^{s} \mathcal{A}$ and for $i<j, \mathrm{t}^{g}\left(a_{i} / \emptyset\right)$ is orthogonal to $\mathrm{t}^{g}\left(a_{j} / \emptyset\right)$. Then the number of models in density character $\omega_{1}$ is $2^{\omega_{1}}$.

Proof. Clearly we may assume that $\mathcal{A}$ is separable. Let $X_{i}, i<2^{\omega_{1}}$ be distinct non-empty subsets of $\omega_{1}$. For all $i<2^{\omega}$ we construct $\mathcal{A}_{i}=\bigcup_{j<\omega_{1}} \mathcal{A}_{i}^{j}$ so that

(1) $\mathcal{A}_{i}^{0}=\mathcal{A}$

(2) for limits $j, \mathcal{A}_{i}^{j}$ is the completion of $\bigcup_{k<j} \mathcal{A}_{i}^{k}$,

(3) $\mathcal{A}_{i}^{j+1}$ is a separable, constructible, complete, $\omega$ - $\mathbf{d}^{p}$-saturated set over $a_{i}^{j} \mathcal{A}_{i}^{j}$, where $a_{i}^{j}$ is such that $\mathrm{t}^{g}\left(a_{i}^{j} / \mathcal{A}\right)=\mathrm{t}^{g}\left(a_{k} / \mathcal{A}\right)$ for some $k \in X_{i}$ and $a_{i}^{j} \downarrow_{\mathcal{A}}^{s} \mathcal{A}_{i}^{j}$,

(4) for all $k \in X_{i},\left|\left\{j<\omega_{1}: \mathrm{t}^{g}\left(a_{i}^{j} / \mathcal{A}\right)=\mathrm{t}^{g}\left(a_{k} / \mathcal{A}\right)\right\}\right|=\omega_{1}$.

By the proof of 7.6, it is easy to see that these models exist.

So we are left to prove that if $i<i^{\prime}$, then $\mathcal{A}_{i}$ is not isomorphic to $\mathcal{A}_{i^{\prime}}$. For a contradiction suppose $f$ is an isomorphism from $\mathcal{A}_{i}$ to $\mathcal{A}_{i^{\prime}}$. Without loss of generality we may assume that there is $k \in X_{i}-X_{i^{\prime}}$. Clearly there is $j<\omega_{1}$ such that $f\left(\mathcal{A}_{i}^{j}\right)=\mathcal{A}_{i^{\prime}}^{j}$. Now choose $j^{\prime} \geq j$ so that $\mathrm{t}^{g}\left(a_{i}^{j^{\prime}} / \mathcal{A}\right)=\mathrm{t}^{g}\left(a_{k} / \mathcal{A}\right)$. An easy induction on $j^{\prime} \leq j^{\prime \prime}<\omega_{1}$ using the dominance shows that $f\left(a_{i}^{j^{\prime}}\right) \downarrow_{\emptyset}^{s}$ $\mathcal{A}_{i^{\prime}}^{j^{\prime \prime}}$ for all $j^{\prime \prime}<\omega_{1}$. This contradicts the fact that $f\left(a_{i}^{j^{\prime}}\right) \in \mathcal{A}_{i^{\prime}}$.

\section{REFERENCES}

[1] I. BEN YAACOV, On perturbations of continuous structures. To appear in the Journal of Mathematical Logic.

[2] I. BEN YAACOV, Topometric spaces and perturbations of metric structures, Log. Anal., 1 (2008), pp. 235-272.

[3] I. Ben YAaCOV And A. Berenstein, On perturbations of Hilbert spaces and probability albegras with a generic automorphism, Journal of Logic and Analysis, 1 (2009), pp. 1-18.

[4] I. Ben YaAcov, A. Berenstein, C. W. Henson, And A. Usvyatsov, Model theory for metric structures, in Model Theory with Applications to Algebra and Analysis, Vol. II, Z. Chatzidakis, D. Macpherson, A. Pillay, and A. Wilkie, eds., vol. 350 of London Math Society Lecture Note Series, Cambridge Univ. Press, Cambridge, 2008, pp. 315-427.

[5] I. Ben YaACOV AND A. Usvyatsov, On d-finiteness in continuous structures, Fundamenta Mathematicae, 194 (2007), pp. 67-88.

[6] I. Ben YaAcov, A. Usvyatsow, And M. ZADKA, Generic automorphism of a Hilbert space. Preprint.

[7] J. M. F. CAstillo And M. GonzÁlez, Three-space problems in Banach space theory, vol. 1667 of Lecture Notes in Mathematics, Springer-Verlag, Berlin, 1997. 
[8] C. W. Henson and J. Iovino, Ultraproducts in analysis, in Analysis and logic (Mons, 1997), vol. 262 of London Math. Soc. Lecture Note Ser., Cambridge Univ. Press, Cambridge, 2002, pp. 1-110.

[9] A. Hirvonen and T. Hyttinen, Categoricity in homogeneous complete metric spaces, Arch. Math. Logic, 48 (2009), pp. 269-322.

[10] T. Hyttinen and S. Shelah, Strong splitting in stable homogeneous models, Ann. Pure Appl. Logic, 103 (2000), pp. 201-228.

[11] J. Iovino, Stable Banach spaces and Banach space structures. I. Fundamentals, in Models, algebras, and proofs (Bogotá, 1995), vol. 203 of Lecture Notes in Pure and Appl. Math., Dekker, New York, 1999, pp. 77-95.

[12] S. Shelah, Classification theory and the number of nonisomorphic models, vol. 92 of Studies in Logic and the Foundations of Mathematics, North-Holland Publishing Co., Amsterdam, second ed., 1990.

[13] S. Willard, General topology, Dover Publications Inc., Mineola, NY, 2004. Reprint of the 1970 original [Addison-Wesley, Reading, MA; MR0264581].

Department of Mathematics and Statistics, University of Helsinki, P.O.Box 68, 00014 University of Helsinki, Finland

E-mail address: asa.hirvonen@helsinki.fi

Department of Mathematics and Statistics, University of Helsinki, P.O.Box 68, 00014 University of Helsinki, Finland

E-mail address: tapani.hyttinen@helsinki.fi 